\title{
Comparative analysis of the diffuse radio emission in the galaxy clusters A1835, A2029, and Ophiuchus
}

\author{
M. Murgia ${ }^{1,2}$, F. Govoni ${ }^{1}$, M. Markevitch ${ }^{3}$, L. Feretti ${ }^{2}$, G. Giovannini ${ }^{2,4}$, G. B. Taylor ${ }^{5, \star}$, and E. Carretti ${ }^{2}$ \\ 1 INAF - Osservatorio Astronomico di Cagliari, Poggio dei Pini, Strada 54, 09012 Capoterra (CA), Italy \\ e-mail: m.murgia@ira.inaf.it \\ 2 INAF - Istituto di Radioastronomia, via Gobetti 101, 40129 Bologna, Italy \\ 3 Harvard-Smithsonian Center for Astrophysics, 60 Garden Street, Cambridge, MA 02138, USA \\ 4 Dipartimento di Astronomia, Univ. Bologna, via Ranzani 1, 40127 Bologna, Italy \\ 5 University of New Mexico, MSC 07 4220, Albuquerque, NM 87131, USA
}

Received 13 January 2009/ Accepted 30 March 2009

ABSTRACT

\begin{abstract}
Aims. We recently performed a study of a sample of relaxed, cooling core galaxy clusters with deep Very Large Array observations at $1.4 \mathrm{GHz}$. We find that in the central regions of A1835, A2029, and Ophiuchus the dominant radio galaxy is surrounded by a diffuse low-brightness radio emission that takes the form of a mini-halo. Here we present the results of the analysis of the extended diffuse radio emission in these mini-halos.

Methods. In order to investigate the morphological properties of the diffuse radio emission in clusters of galaxies we propose to fit their azimuthally averaged brightness profile with an exponential, obtaining the central brightness and the $e$-folding radius from which the radio emissivity can be calculated. We investigate the radio properties of the mini-halos in A1835, A2029, and Ophiuchus in comparison with the radio properties of a representative sample of mini-halos and halos already known in the literature.

Results. We find that radio halos can have quite different length-scales but their emissivity is remarkably similar from one halo to the other. In contrast, mini-halos span a wide range of radio emissivity. Some of them, like the Perseus mini-halo, are characterized by a radio emissivity which is more than 100 times greater than that of radio halos. On the other hand, the new mini-halos in cooling core clusters analyzed in this work, namely A2029, Ophiuchus, and A1835, have a radio emissivity which is much more typical of halos in merging clusters rather than similar to that of the other mini-halos previously known.
\end{abstract}

Key words. galaxies: clusters: individual: A1835, A2029, Ophiuchus - radio continuum: galaxies

\section{Introduction}

Radio halos are diffuse, low surface brightness, steep-spectrum ${ }^{1}$ sources $(\alpha \gtrsim 1)$, permeating the central regions of galaxy clusters. These radio sources are extended on megaparsec scales and are produced by synchrotron radiation of relativistic electrons with energies of $\simeq 10 \mathrm{GeV}$ in magnetic fields with $B \simeq 0.5-1 \mu \mathrm{G}$ (see e.g. Feretti \& Giovannini 2008; Ferrari et al. 2008, and references therein for recent reviews).

Radio halos are typically found in clusters which show significant evidence for an ongoing merger (e.g. Buote 2001; Govoni et al. 2004). It has been proposed that recent cluster mergers may play an important role in the re-acceleration of the radio-emitting relativistic particles, thus providing the energy that powers these extended sources (e.g. Brunetti et al. 2001; Petrosian 2001). A major merger event is expected to disrupt cooling cores and create disturbances readily visible in an X-ray image of the cluster. Therefore, the merger scenario predicts the absence of large-scale radio halos in symmetric cooling core clusters. However, a few cooling core clusters show the presence of a diffuse synchrotron emission that extends quite far from the dominant radio galaxy at the cluster center, forming what is called a mini-halo. These diffuse radio sources are extended on a moderate scale (typically $\simeq 500 \mathrm{kpc}$ ) and, in common with

* G.B. Taylor is also an Adjunct Astronomer at the National Radio Astronomy Observatory

${ }^{1}$ We use the convention $S_{v} \propto v^{-\alpha}$ large-scale halos, have a steep spectrum and a very low surface brightness.

To date about 30 radio halos are known (e.g., Giovannini \& Feretti 2000; Bacchi et al. 2003; Govoni et al. 2001a; Venturi et al. 2007, 2008; Giovannini et al., in preparation). Due to their extremely low surface brightness and large angular extent $\left(>10^{\prime}\right.$ at a redshift $\left.z \leq 0.1\right)$ radio halos are best studied at low spatial resolution. In fact, several radio halos were detected by Giovannini et al. (1999) in the NRAO VLA Sky Survey (NVSS, Condon et al. 1998) and by Kempner \& Sarazin (2001) in the Westerbork Northern Sky Survey (WENSS; Rengelink et al. 1997), where the relatively large beam of these surveys provided the necessary sensitivity to large-scale emission to spot these elusive sources.

Due to the combination of their small angular size and the strong radio emission of the central radio galaxy, the detection of a mini-halo requires a much higher dynamic range and resolution than provided by available surveys, thus complicating their detection. As a consequence, our current observational knowledge on mini-halos is limited to only a handful of well-studied clusters (e.g., Perseus: Burns et al. 1992; A2390: Bacchi et al. 2003; RXJ1347.5-1145: Gitti et al. 2007), and their origin and physical properties are still poorly understood.

To search for new extended diffuse radio emission in relaxed and cooling core galaxy clusters, we recently performed a study of a small sample of clusters with deep Very Large Array observations at $1.4 \mathrm{GHz}$. We find that in the central regions of 
Table 1. The mini-halos analyzed in this work and the basic properties of the radio images at $1.4 \mathrm{GHz}$.

\begin{tabular}{ccccc}
\hline \hline Cluster & $z$ & $\mathrm{kpc} /{ }^{\prime \prime}$ & Beam & $\begin{array}{c}\sigma \\
\mathrm{mJy} / \text { beam }\end{array}$ \\
\hline A1835 & 0.2532 & 3.91 & $53^{\prime \prime} \times 53^{\prime \prime}$ & 0.025 \\
A2029 & 0.0765 & 1.43 & $53^{\prime \prime} \times 53^{\prime \prime}$ & 0.031 \\
Ophiuchus & 0.028 & 0.55 & $92^{\prime \prime} \times 92^{\prime \prime}$ & 0.16 \\
\hline
\end{tabular}

Column 3: Angular to linear size conversion factor; Col. 4: FWHM beam of the radio images; Col. 5: image sensitivity.

A1835, A2029, and Ophiuchus the dominant radio galaxy is surrounded by a diffuse low-brightness radio emission that takes the form of a mini-halo (Govoni et al. 2009, hereafter Paper I). Here we present the analysis of these three new mini-halos and we investigate their radio properties in comparison with those of a statistically significant sample of mini-halos and halos already known in the literature and for which good VLA radio images at $1.4 \mathrm{GHz}$ are available. In Sect. 2 we present an analysis of the mini-halos found in A1835, A2029, and Ophiuchus. In particular we fit their azimuthally averaged brightness profile with an exponential. Through the study of their averaged brightness profiles, we analyze some of their morphological and physical radio properties (i.e., length-scale, central brightness, and emissivity). In Sect. 3 we compare the properties of the mini-halos in A1835, A2029, and Ophiuchus with those of other halos and mini-halos already available in the literature. In Sect. 4 we test the reliability of the fitting procedure. The discussion is presented in Sect. 5 and our conclusions are reported in Sect. 6.

Throughout this paper we assume a $\Lambda \mathrm{CDM}$ cosmology with $H_{0}=71 \mathrm{~km} \mathrm{~s}^{-1} \mathrm{Mpc}^{-1}, \Omega_{\mathrm{m}}=0.27$, and $\Omega_{\Lambda}=0.73$.

\section{Analysis of the mini-halos in A1835, A2029 and Ophiuchus}

In order to investigate the presence of diffuse extended emission in relaxed systems, we recently examined a small sample of cooling core clusters with deep Very Large Array observations at $1.4 \mathrm{GHz}$. The combination of resolution and sensitivity of these observations allow us to increase the number of known large scale diffuse radio emissions in cooling core clusters. Indeed, we found that in the central regions of A1835, A2029, and Ophiuchus the dominant radio galaxy is surrounded by a diffuse low-brightness radio emission that takes the form of a mini-halo. The basic properties of these clusters are reported in Table 1, while the details of the observations are described in Paper I. Here we present a morphological analysis of these mini-halos. For this purpose, the radio images have been convolved with a circular Gaussian beam and corrected for the primary beam attenuation.

The brightness of halo-like diffuse radio sources in clusters of galaxies decreases with increasing distance from the cluster center, eventually falling below the noise level of the radio images. The signal-to-noise ratio of the VLA images analyzed in Paper I allows us to trace the mini-halo extension out to a distance from the cluster center of $\sim 450 \mathrm{kpc}, \sim 250 \mathrm{kpc}$, and $200 \mathrm{kpc}$ for A1835, A2029, and Ophiuchus, respectively (see left-panels of Fig. 1). However, the size of the diffuse lowsurface brightness emissions as derived from the $3 \sigma$-isophotes seen in the radio images may be strongly affected by the sensitivity of the radio observations. It is desirable to define a quantity which is independent of the signal-to-noise ratio of the radio images in order to obtain an unbiased estimate of the size of mini-halos and halos.

Although deviations of the diffuse emission from spherical symmetry are often observed, the azimuthally averaged radial profiles are indeed quite stable. For this reason, following the same approach used by Orrù et al. (2007), we fit the azimuthally averaged brightness profiles with an exponential law of the form:

$I(r)=I_{0} \mathrm{e}^{-r / r_{e}}$

where the two independent parameters $I_{0}$ and $r_{e}$ are the central radio surface brightness and the $e$-folding radius i.e., the radius at which the brightness drops to $I_{0} / e$. As we will show, the quantity $r_{e}$ can be used to define a length-scale which is relatively independent on the sensitivity of the radio images. The exponential model is attractive in its simplicity and involves a minimal set of free parameters.

We note that in mini-halos the presence of a strong central radio galaxy, which appears as a point source at the low resolution of our observations, complicates the analysis. Therefore its emission must be known accurately in order to separate its contribution from that of the mini-halo.

In Fig. 1 we present the resulting azimuthally averaged brightness profiles of A1835, A2029 and Ophiuchus, respectively. The profiles have been obtained by averaging the radio brightness in concentric annuli as shown in the left panels of Fig. 1. The annuli are as wide as the half $F W H M$ beam. In the statistical analysis all the discrete sources (except the central source) have been excluded by masking them out. Moreover, we considered only data points above the 3 -sigma radio noise level as indicated by the horizontal dashed-dotted line in the plots.

In order to carefully separate the contribution of the minihalo from that of the central radio galaxy, we fitted the total brightness profiles with a central point source plus the radio mini-halo diffuse emission

$I(r)=I_{\mathrm{PS}}(r)+I_{\mathrm{MH}}(r)$.

In particular the profile of the central point source has been characterized by a Gaussian of the form

$I_{\mathrm{PS}}(r)=I_{0_{\mathrm{PS}}} \mathrm{e}^{-\left(r^{2} / 2 \sigma_{\mathrm{PS}}^{2}\right)}$

while the brightness profile of the mini-halo has been characterized by an exponential law of the form

$I_{\mathrm{MH}}(r)=I_{0_{\mathrm{MH}}} \mathrm{e}^{-r / r_{e}}$.

The resolution of the observations, the masked regions, and the sampling of the radial profile in annuli, are all factors that can affect the estimate of the best fit parameters and thus we decided to include their effects directly in the modelling.

In order to properly take into account the resolution, the exponential model in Eq. (4) is first calculated in a 2-dimensional image with the same pixel size and field of view as that observed, and then convolved with the same beam by means of a Fast Fourier Transform. The point-source model in Eq. (3) is added and the resulting image is masked exactly in the same regions as for the observations. Finally, the model is azimuthally averaged with the same set of annuli used to obtain the observed radial profile. All these functions are performed at each step during the fit procedure. As a result, the values of the central brightness, $I_{0}$, and $e$-folding radius, $r_{e}$, provided by the fit are deconvolved quantities and their estimate includes all the uncertainties related to the masked regions and to the sampling of the radial profile in 

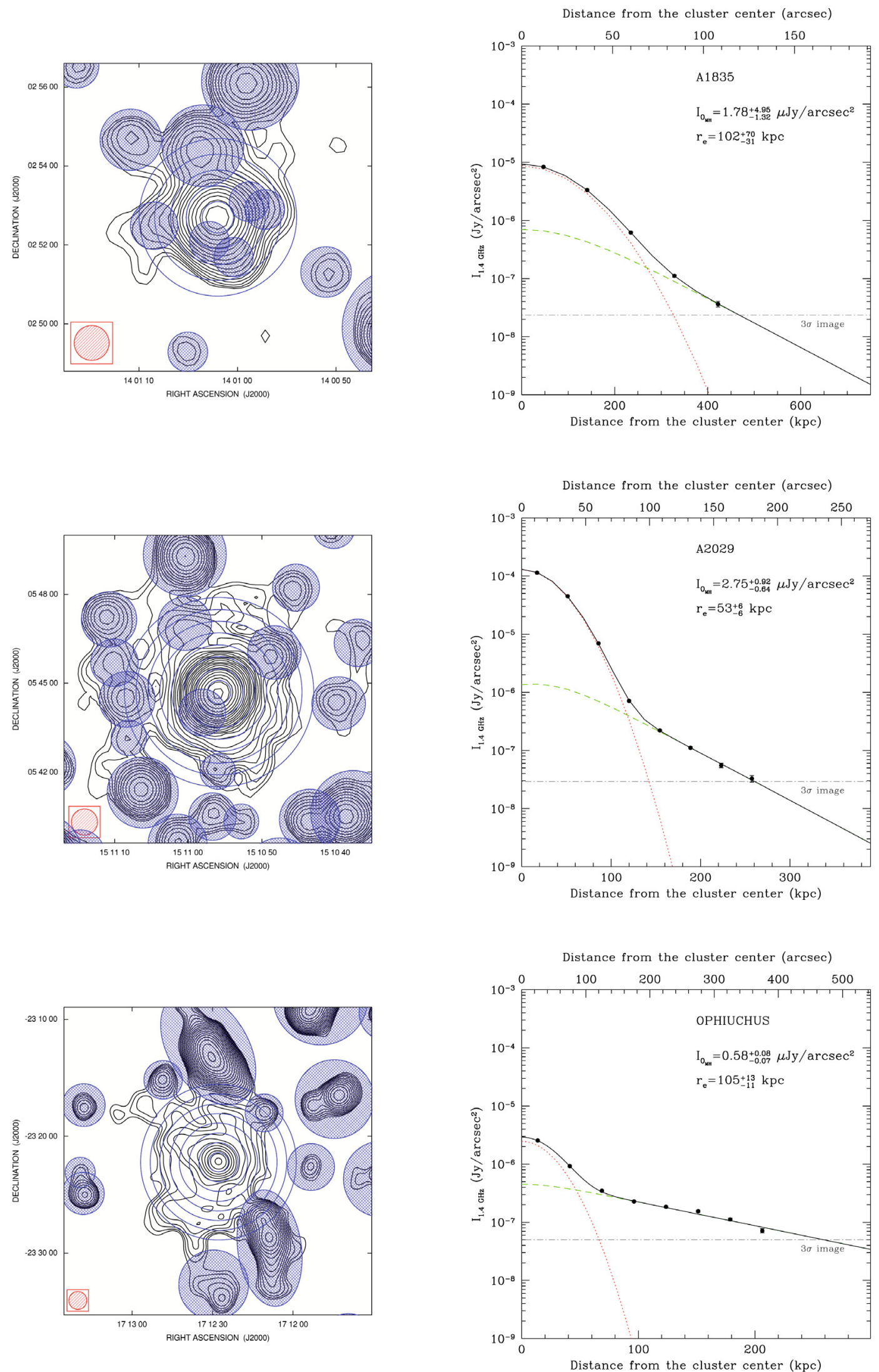

Fig. 1. The azimuthally averaged brightness profiles of the radio emission in A1835 (top), A2029 (middle), and Ophiuchus (bottom). The profiles have been calculated in concentric annuli, as shown in the left panels. All the discrete sources (except the central source) have been masked. For each cluster the horizontal dashed-dotted line indicates the $3 \sigma$ noise level of the radio image. In our analysis we considered all the data points above the $3 \sigma$ noise level. The black line indicates the best fit profile described by an exponential law (dashed line) representing the mini-halo emission, and by a central Gaussian profile (dotted line) representing the central point source. 
Table 2. Radio properties of the mini halos derived from the fit procedure.

\begin{tabular}{|c|c|c|c|c|c|c|c|c|c|c|}
\hline Cluster & $\begin{array}{c}F W H M \\
\operatorname{arcsec}\end{array}$ & $\begin{array}{c}r_{e} \\
\operatorname{arcsec}\end{array}$ & $\begin{array}{c}r_{e} \\
\mathrm{kpc}\end{array}$ & $\begin{array}{c}I_{0_{\mathrm{MH}}} \\
\mu \mathrm{Jy} / \operatorname{arcsec}^{2}\end{array}$ & $\begin{array}{l}S_{\mathrm{MH}} \\
\mathrm{mJy}\end{array}$ & $\begin{array}{c}I_{0_{\mathrm{PS}}} \\
\mu \mathrm{Jy} / \operatorname{arcsec}^{2}\end{array}$ & $\begin{array}{c}F W H M_{\mathrm{PS}} \\
\operatorname{arcsec}\end{array}$ & $\begin{array}{l}S_{\mathrm{PS}} \\
\mathrm{mJy}\end{array}$ & $\begin{array}{c}\langle\overline{\langle J\rangle} \\
\mathrm{erg} \mathrm{s}^{-1} \mathrm{~Hz}^{-1} \mathrm{~cm}^{-3}\end{array}$ & $\overline{\chi_{\mathrm{RED}}^{2}}$ \\
\hline A1835 & 53 & $26_{-8}^{+18}$ & $102_{-31}^{+70}$ & $1.78_{-1.32}^{+4.95}$ & $6.0_{-0.7}^{+0.8}$ & $8.68_{-1.11}^{+0.65}$ & $57_{-3}^{+2}$ & $32.2_{-0.7}^{+0.8}$ & $3.3_{-1.3}^{+2.2} \times 10^{-42}$ & 0.03 \\
\hline A2029 & 53 & $37_{-4}^{+4}$ & $53_{-6}^{+6}$ & $2.75_{-0.64}^{+0.92}$ & $18.8_{-1.1}^{+1.3}$ & $129_{-7}^{+7}$ & $57_{-1}^{+1}$ & $480.0_{-19.4}^{+19.5}$ & $5.4_{-1.3}^{+1.9} \times 10^{-42}$ & 0.5 \\
\hline OPHIUCHUS & 92 & $191_{-19}^{+23}$ & $105_{-11}^{+13}$ & $0.58_{-0.07}^{+0.08}$ & $106.4_{-8.9}^{+10.4}$ & $2.47_{-0.21}^{+0.22}$ & $102_{-5}^{+6}$ & $29.0_{-2.0}^{+2.0}$ & $4.7_{-0.8}^{+0.9} \times 10^{-43}$ & 2.9 \\
\hline
\end{tabular}

Column 2: FWHM of the circular Gaussian beam; Col. 6: mini-halo radio flux density calculated up to $3 r_{e}$; Col. 10: average radio emissivity over the volume of a sphere of radius $3 r_{e}, k$-corrected with $\alpha=1$.

annuli of finite width. The fit procedure has been implemented in the software FARADAY (Murgia et al. 2004).

The best fit model is shown as a continuous black line in the right panels of Fig. 1. The mini-halo contribution is indicated by the green dashed line, while the contribution of the central point source is indicated with the red dotted line. The best fit values for both the mini-halos and the central components are reported in Table 2, together with their respectively errors and the corresponding $\chi^{2}$.

Overall, the observed profiles are well fit by the exponential model. In A2029 and Ophiuchus the emission of the minihalo extends for several beams from the central point source. It is important to note the fundamental difference between the method based on the measurement of $r_{\max }$, the mini-halo maximum size from the $3 \sigma$-isophote above the noise ${ }^{2}$, and the fit of the $e$-folding radius, $r_{e}$. In fact, the mini-halo in A2029 has a larger extension with respect to that in Ophiuchus: the two mini-halos have $r_{\max } \sim 250 \mathrm{kpc}$ and $r_{\max } \sim 200 \mathrm{kpc}$, respectively. However, the mini-halo emission in A2029 falls off more rapidly and the corresponding $e$-folding radius $r_{e}=53_{-6}^{+6} \mathrm{kpc}$ is much smaller than that in Ophiuchus, $r_{e}=105_{-11}^{+13} \mathrm{kpc}$. The fit is critical for A1835 (see Sect. 4). This is the most distant cluster of the three and the emission of the mini-halo is heavily blended with that of the central point source. The maximum extent for A1835 is as large as $r_{\max } \sim 450 \mathrm{kpc}$, while the $e$-folding radius obtained by the model fit results, with a large uncertainty, is $r_{e}=105_{-21}^{+70} \mathrm{kpc}$.

The central brightness ranges from $I_{0}=2.75 \mu \mathrm{Jy} / \mathrm{arcsec}^{2}$ for A2029 to $I_{0}=0.58 \mu \mathrm{Jy} / \operatorname{arcsec}^{2}$ for Ophiuchus, indicating a slightly tendency for the smaller mini-halos to have a higher central brightness (see Sect. 3). In all three cases the best fit model results in a central Gaussian with $F W H M_{\mathrm{PS}}=\sigma_{\mathrm{PS}} \times 2.35 \gtrsim$ $F W H M_{\text {beam }}$, as expected for a point like or a slightly resolved source. From $I_{0}$ and $r_{e}$ we then calculated the flux density $S_{\mathrm{MH}}$ (see Table 2) of the mini-halos. In particular, the flux density is derived by integrating the brightness profile $I(r)$ up to a radius $r^{\prime}$ from the cluster center:

$S_{\mathrm{MH}}=2 \pi \int_{0}^{r^{\prime}} I(r) r \mathrm{~d} r=2 \pi\left[1+\mathrm{e}^{-r^{\prime} / r_{e}}\left(-r^{\prime} / r_{e}-1\right)\right] \cdot r_{e}^{2} I_{0}$.

We calculated the flux density of the mini-halos by integrating the surface brightness of the best fit exponential model up to $r^{\prime}=3 r_{e}$, giving:

$S_{\mathrm{MH}}=2 \pi f \cdot r_{e}^{2} I_{0} \quad(\mathrm{Jy})$

where $r_{e}$ and $I_{0}$ are in units of $\operatorname{arcsec}$ and Jy/arcsec ${ }^{2}$, respectively, while $f=\left(1-4 e^{-3}\right) \simeq 0.8$. Thus, the flux density in Eq. (6) consists of about $80 \%$ of the total model flux that would be obtained

2 In this paper, we define $r_{\max }$ as the distance from the cluster center at which the azimuthally averaged brightness profile of the diffuse emission reaches the $3 \sigma$ noise level of the radio image. by extrapolating $r^{\prime}$ to $+\infty$. It must be noted that the model flux calculated from Eq. (6) may differ from the flux density measured by integrating the radio brightness up to the $3 \sigma$-isophote. For instance, in the case of Ophiuchus $r_{\max }<3 r_{e}$ and the model flux of $106 \mathrm{mJy}$ is higher than the flux density of about $80 \mathrm{mJy}$ which is obtained by integrating up to the $3 \sigma$-isophote. Instead, in the case of A2029, $r_{\max }>3 r_{e}$ and the model flux density of $18.8 \mathrm{mJy}$ is lower than the flux density of $22.5 \mathrm{mJy}$ obtained by integrating up to the $3 \sigma$-isophote.

In Col. 9 of Table 2, we also provide the flux density of the central point source derived from the fit. The flux density of the central point source, $S_{\mathrm{PS}}$, is calculated through:

$S_{\mathrm{PS}}=\frac{2 \pi}{8 \ln (2)} \cdot F W H M_{\mathrm{PS}}^{2} I_{0_{\mathrm{PS}}} \quad(\mathrm{Jy})$

where $F W H M_{\mathrm{PS}}$ is measured in arcsec and $I_{0_{\mathrm{PS}}}$ in Jy/arcsec ${ }^{2}$. Since mini-halos are optically thin sources, the radio brightness $I$, at a projected distance $r$ from the cluster centre, is related to the radio emissivity $J(R)$ through the line-of-sight integral:

$I(r)=\frac{1}{4 \pi} \int_{\text {source }} J(R) \mathrm{d} l$

where $R=\sqrt{r^{2}+l^{2}}$.

We calculated a volume-averaged radio emissivity by supposing that all the flux density in Eq. (6) comes from a sphere of radius $3 r_{e}$ :

$\langle J\rangle \simeq 7.7 \times 10^{-41}(1+z)^{3+\alpha} \cdot \frac{I_{0}}{r_{e}} \quad\left(\mathrm{erg} \mathrm{s}^{-1} \mathrm{~cm}^{-3} \mathrm{~Hz}^{-1}\right)$

where $r_{e}$ and $I_{0}$ are in units of $\mathrm{kpc}$ and $\mu \mathrm{Jy} / \operatorname{arcsec}^{2}$, respectively. The factor $(1+z)^{3+\alpha}$ takes into account of both the $k$-correction and cosmological dimming of the surface brightness with redshift. The radio emissivity for the three mini-halos $(k$-corrected with a spectral index $\alpha=1$ ) is reported in Col. 10 of Table 2.

\section{Mini-halos and halos comparison}

Merging and cooling core clusters containing extended diffuse radio sources (halos and mini-halos respectively) show different X-ray properties, indicating a different evolutionary state. A systematic and homogeneous comparison between the properties of extended diffuse radio sources in merging systems with those in cooling core clusters, may help in understanding if the energy necessary to trigger their radio emission may be related to different physical processes. Therefore, we analyzed the azimuthally averaged brightness profiles in a sample of 12 clusters containing a central radio halo, previously imaged by us. Moreover, in order to investigate also a significant number of mini-halos, we reduced and analyzed the VLA data at $1.4 \mathrm{GHz}$ of the mini-halos in RXJ1347.5-1145 (Gitti et al. 2007), A2390 
Table 3. Radio information of halos and mini halos taken from the literature and reanalyzed in this work with the exponential model fit.

\begin{tabular}{|c|c|c|c|c|c|c|c|c|c|c|}
\hline Cluster & Type & Reference & $z$ & $\mathrm{kpc} /{ }^{\prime \prime}$ & $\begin{array}{c}F W H M \\
\operatorname{arcsec}\end{array}$ & $\begin{array}{c}I_{0} \\
\mu \mathrm{Jy} / \operatorname{arcsec}^{2}\end{array}$ & $\begin{array}{c}r_{e} \\
\operatorname{arcsec}\end{array}$ & $\begin{array}{r}r_{e} \\
\mathrm{kpc}\end{array}$ & $\begin{array}{c}\langle J\rangle \\
\operatorname{erg~s}^{-1} \mathrm{~Hz}^{-1} \mathrm{~cm}^{-3}\end{array}$ & $\chi_{\mathrm{RED}}^{2}$ \\
\hline A2744 & $\mathrm{H}$ & Govoni et al. (2001a) & 0.3080 & 4.50 & 50 & $3.05_{-0.12}^{+0.12}$ & $61_{-2}^{+2}$ & $275_{-9}^{+9}$ & $2.5_{-0.16}^{+0.17} \times 10^{-42}$ & 1.5 \\
\hline A665 & $\mathrm{H}$ & Giovannini Feretti (2000) & 0.1819 & 3.03 & 53 & $1.09_{-0.08}^{+0.08}$ & $78_{-5}^{+6}$ & $236_{-15}^{+18}$ & $7.0_{-1.0}^{+1.0} \times 10^{-43}$ & 0.9 \\
\hline A2219 & $\mathrm{H}$ & Bacchi et al. (2003) & 0.2256 & 3.59 & 53 & $1.10_{-0.08}^{+0.08}$ & $100_{-4}^{+5}$ & $359_{-14}^{+18}$ & $5.4_{-0.6}^{+0.6} \times 10^{-43}$ & 3.7 \\
\hline A2255 & $\mathrm{H}$ & Govoni et al. (2005) & 0.0806 & 1.50 & 25 & $0.65_{-0.02}^{+0.02}$ & $135_{-4}^{+4}$ & $203_{-6}^{+6}$ & $3.4_{-0.19}^{+0.20} \times 10^{-43}$ & 4.8 \\
\hline A773 & $\mathrm{H}$ & Govoni et al. (2001a) & 0.2170 & 3.48 & 30 & $0.75_{-0.07}^{+0.07}$ & $32_{-3}^{+3}$ & $111_{-10}^{+10}$ & $1.1_{-0.2}^{+0.24} \times 10^{-42}$ & 0.7 \\
\hline A545 & $\mathrm{H}$ & Bacchi et al. (2003) & 0.1540 & 2.64 & 45 & $1.31_{-0.13}^{+0.15}$ & $57_{-4}^{+4}$ & $150_{-11}^{+11}$ & $1.2_{-0.19}^{+0.22} \times 10^{-42}$ & 0.6 \\
\hline A2319 & $\mathrm{H}$ & Feretti et al. (1997) & 0.0557 & 1.07 & 30 & $1.11_{-0.04}^{+0.04}$ & $185_{-6}^{+7}$ & $198_{-6}^{+7}$ & $5.4_{-0.36}^{+0.35} \times 10^{-43}$ & 2.4 \\
\hline A2218 & $\mathrm{H}$ & Giovannini Feretti (2000) & 0.1756 & 2.94 & 35 & $1.06_{-0.27}^{+0.34}$ & $26_{-6}^{+9}$ & $76_{-18}^{+26}$ & $2.0_{-0.9}^{+1.4} \times 10^{-42}$ & 1.0 \\
\hline A 2163 & $\mathrm{H}$ & Feretti et al. (2001) & 0.2030 & 3.31 & 62 & $2.23_{-0.07}^{+0.07}$ & $119_{-2}^{+2}$ & $394_{-7}^{+7}$ & $9.2_{-0.4}^{+0.4} \times 10^{-43}$ & 2.1 \\
\hline A401 & $\mathrm{H}$ & Bacchi et al. (2003) & 0.0737 & 1.38 & 45 & $0.44_{-0.05}^{+0.06}$ & $79_{-11}^{+15}$ & $109_{-15}^{+21}$ & $4.1_{-1.0}^{+1.3} \times 10^{-43}$ & 0.7 \\
\hline A2254 & $\mathrm{H}$ & Govoni et al. (2001a) & 0.1780 & 2.98 & 45 & $1.56_{-0.20}^{+0.22}$ & $80_{-13}^{+19}$ & $238_{-39}^{+57}$ & $9.7_{-2.8}^{+3.4} \times 10^{-43}$ & 1.1 \\
\hline RXJ1314 & $\mathrm{H}$ & Feretti et al. (2005) & 0.2439 & 3.81 & 45 & $1.05_{-0.25}^{+0.31}$ & $42_{-10}^{+16}$ & $160_{-38}^{+61}$ & $1.2_{-0.5}^{+0.8} \times 10^{-42}$ & 0.1 \\
\hline RXJ1347 & MH & Gitti et al. (2007) & 0.4510 & 5.74 & 18 & $26.0_{-15.4}^{+40.7}$ & $9_{-2}^{+3}$ & $52_{-11}^{+17}$ & $1.8_{-0.5}^{+0.7} \times 10^{-40}$ & 0.2 \\
\hline A2390 & MH & Bacchi et al. (2003) & 0.2280 & 3.62 & 20 & $60.8_{-21.1}^{+27.0}$ & $10_{-1}^{+1}$ & $36_{-4}^{+4}$ & $3.1_{-0.8}^{+1.0} \times 10^{-40}$ & 1.2 \\
\hline Perseus & MH & Pedlar et al. (1990) & 0.0179 & 0.36 & 45 & $99.6_{-106}^{+10.9}$ & $64_{-3}^{+4}$ & $23_{-1}^{+1}$ & $3.6_{-0.5}^{+0.5} \times 10^{-40}$ & 2.4 \\
\hline
\end{tabular}

Column 2: type of diffuse emission contained ( $\mathrm{H}=$ halo, $\mathrm{MH}=$ mini halo); Col. 6: FWHM of the circular Gaussian beam; Col. 10: average radio emissivity over the volume of a sphere of radius $3 r_{e}, k$-corrected with $\alpha=1$.

(Bacchi et al. 2003), and Perseus (Pedlar et al. 1990), previously reported in the literature. The cluster list, together with their references is given in Table 3.

Indeed the analysis lacks some well known radio halos and mini-halos, however, the number of clusters analyzed here is representative of these classes of radio sources and contains both extended (e.g. A2163, A2744) and small halos (e.g. A401, A2218) in merging clusters and several mini-halos in cooling core clusters. In Figs. 2 and 3 we present the resulting azimuthally averaged brightness profiles of the radio halos, while in Fig. 4 we present those of the three mini-halos. The profiles have been obtained by averaging the radio brightness in concentric annuli as shown in the inset of each panel.

The analysis of the three previously known mini-halos has been performed with the same approach proposed for A1835, A2029, and Ophiuchus - by fitting the brightness profiles with a central Gaussian plus an exponential law, and by masking all the discrete sources, except the central one. The analysis of the halos has been performed by fitting the brightness profiles with only an exponential law and by masking all the discrete sources. As in the case of A1835, A2029, and Ophiuchus the diffuse synchrotron emission both of the halos and mini-halos are quite well fit by an exponential law characterized by a central surface brightness $I_{0}$ and an $e$-folding radius $r_{e}$. The best fit values of the fit procedure, together with their respectively errors, and the corresponding $\chi^{2}$, are reported in Table 3 .

In the top panel of Fig. 5 we show the best fit central brightness $I_{0}\left(\mu \mathrm{Jy} / \operatorname{arcsec}^{2}\right)$ versus the length-scale $r_{e}(\mathrm{kpc})$ both for halos (blue triangles) and mini-halos (red dots). The length scale $r_{e}$ in this sample of halos ranges between $\simeq 70-400 \mathrm{kpc}$. While the central brightness $I_{0}$ ranges between $\simeq 0.4-3 \mu \mathrm{Jy} / \mathrm{arcsec}^{2}$. All the halo sources of the sample populate a well defined region of the $I_{0}-r_{e}$ plane. There is a tendency for halo sources with a higher central brightness to have a greater length-scale. By comparing the location of the halo sources in the $I_{0}-r_{e}$ plane with the dotted lines of constant emissivity we found, for the first time, that all halos have a markedly similar radio emissivity ${ }^{3}$.

In contrast, the mini-halo distribution in the $I_{0}-r_{e}$ plane appears much more scattered. In our sample there are no minihalos with a $r_{e}$ larger than $\simeq 100 \mathrm{kpc}$. They have a tendency to populate the low $r_{e}$, while the central emissivity can be two orders of magnitude higher than in halos. By comparing the location of the mini-halo sources in the $I_{0}-r_{e}$ plane with the lines of constant emissivity we found that although some mini-halos (like Ophiuchus and A1835) are quite comparable to the halo sources, in general mini-halos appear clearly separated from the halo sources, showing a larger spread in radio emissivity.

As previously noted, there is a tendency for the smaller minihalos of our sample to have a higher central brightness. But this trend must be considered carefully, because a selection effect may be present. The dashed line in top panel of Fig. 5 indicates the minimum central brightness required for a mini-halo (or a halo) with a given $e$-folding radius to be imaged with at least 4 resolution elements (beams) in a deep image with a sensitivity of $\sigma=25 \mu \mathrm{Jy} / \mathrm{beam}$, and a $25 \mathrm{arcsec}$ beam. These numbers are appropriate for the deepest images taken with the VLA at this resolution and frequency (see e.g. Govoni et al. 2005). The detection limit is calculated assuming a putative redshift of $z=0.18$ (the average redshift of the mini-halos and halos analyzed in this work). In practice, for a mini-halo (or a halo) to be detected, it is required that $r_{\max }>2 \cdot F W H M_{\text {beam }}$, where $r_{\max }$ is implicitly defined by the equation: $I_{0} \exp \left(-r_{\max } / r_{e}\right)=3 \sigma$ which implies $I_{0} \geq 3 \sigma \exp \left(2 \cdot F W H M_{\text {beam }} / r_{e}\right)$. The shaded region limited by this detection threshold is hardly accessible by current radio interferometers at $1.4 \mathrm{GHz}$. For instance, an object with $r_{e} \simeq 50 \mathrm{kpc}$ would be detectable only if brighter

3 However, we cannot exclude the possibility that large halos with faint surface brightness $\left(I_{0}<0.5 \mu \mathrm{Jy} / \operatorname{arcsec}^{2}\right)$ could have been missed by the current searches at $1.4 \mathrm{GHz}$ such that we are just seeing the "tip of the iceberg". As an example, the $3 \sigma$ sensitivity level of the NVSS is indicated by the dot-dashed line in the top panel of Fig. 5 . 

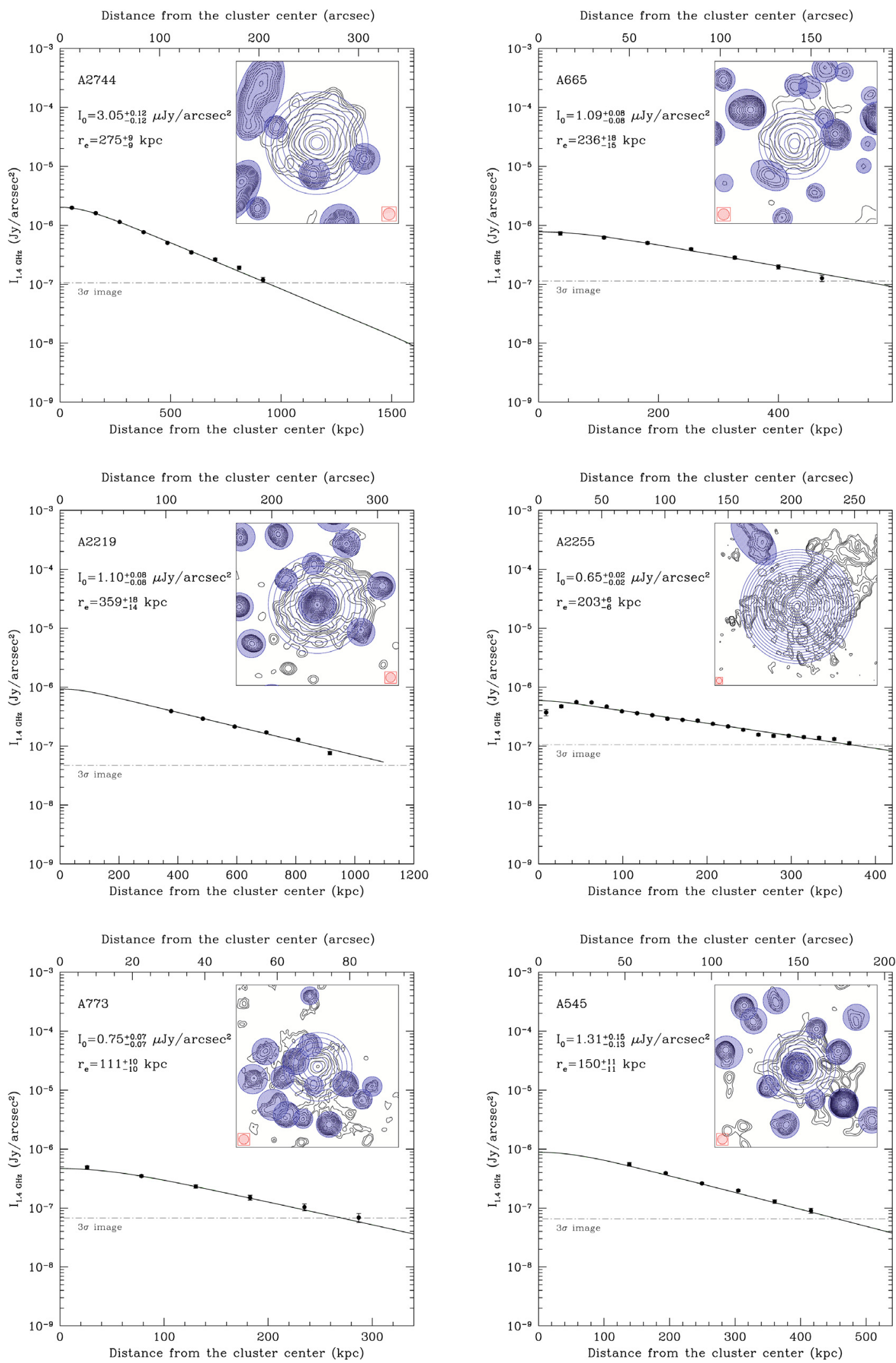

Fig. 2. Azimuthally averaged brightness profiles of the radio halos in A2744 (Govoni et al. 2001a), A665 (Giovannini \& Feretti 2000 ), A2219 (Bacchi et al. 2003), A2255 (Govoni et al. 2005), A773 (Govoni et al. 2001a), A545 (Bacchi et al. 2003). The profiles have been calculated in concentric annuli, as shown in the inset panels. All the discrete sources have been masked. For each cluster the horizontal dashed-dotted line indicates the $3 \sigma$ noise level of the radio image. In the analysis we considered all the data points above the $3 \sigma$ radio noise level. The black line indicates the best fit exponential profile. 
M. Murgia et al.: Comparative analysis of the diffuse radio emission in the galaxy clusters A1835, A2029, and Ophiuchus
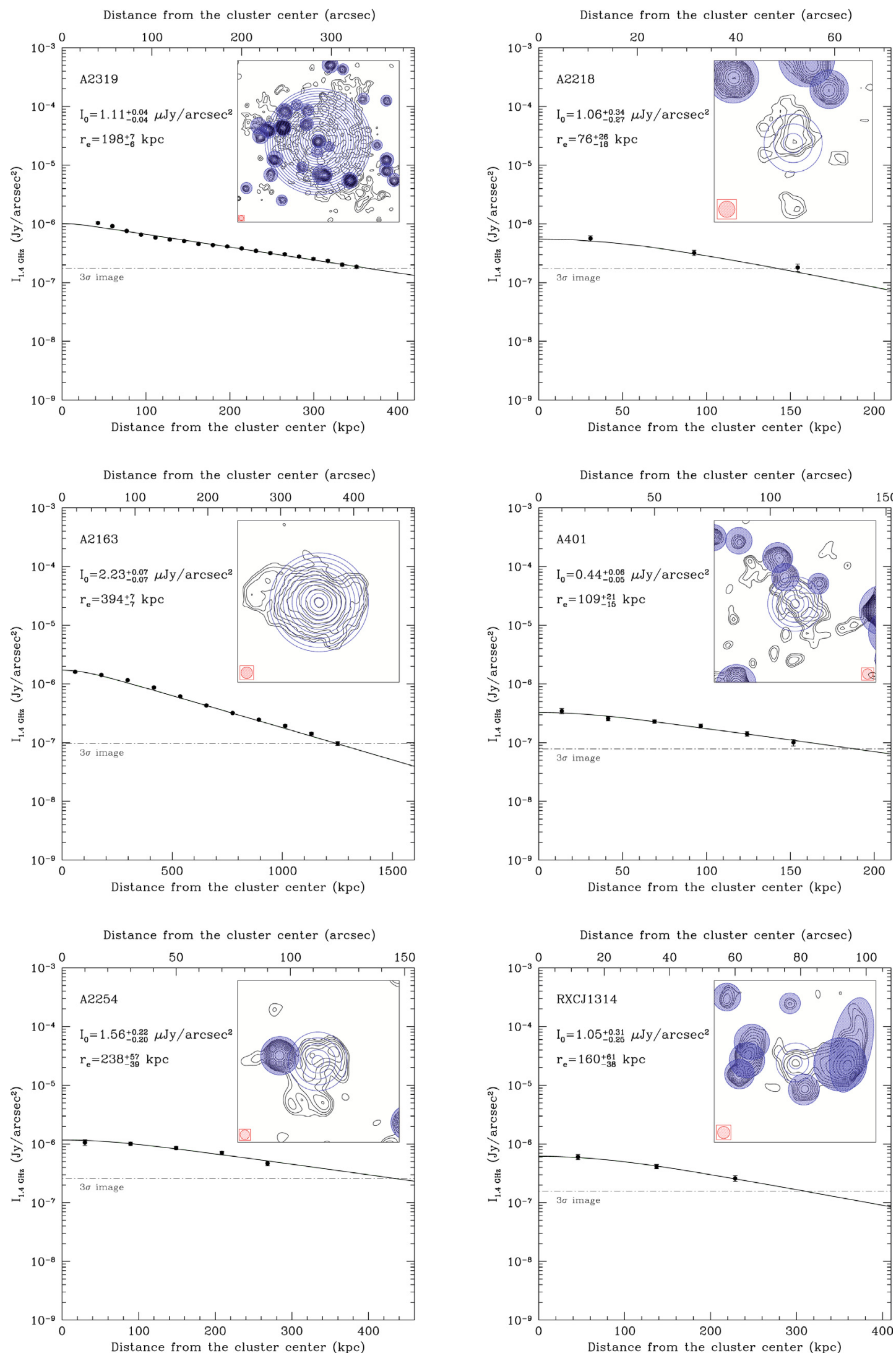

Fig. 3. Azimuthally averaged brightness profiles of the radio halos in A2319 (Feretti et al. 1997), A2218 (Giovannini \& Feretti 2000 ), A2163 (Feretti et al. 2001), A401 (Bacchi et al. 2003), A2254 (Govoni et al. 2001a), RXCJ1314.4-2515 (Feretti et al. 2005). The profiles have been calculated in concentric annuli, as shown in the inset panels. All the discrete sources have been masked. For each cluster the horizontal dasheddotted line indicates the $3 \sigma$ noise level of the radio image. In the analysis we considered all the data points above the $3 \sigma$ radio noise level. The black line indicates the best fit exponential profile. 

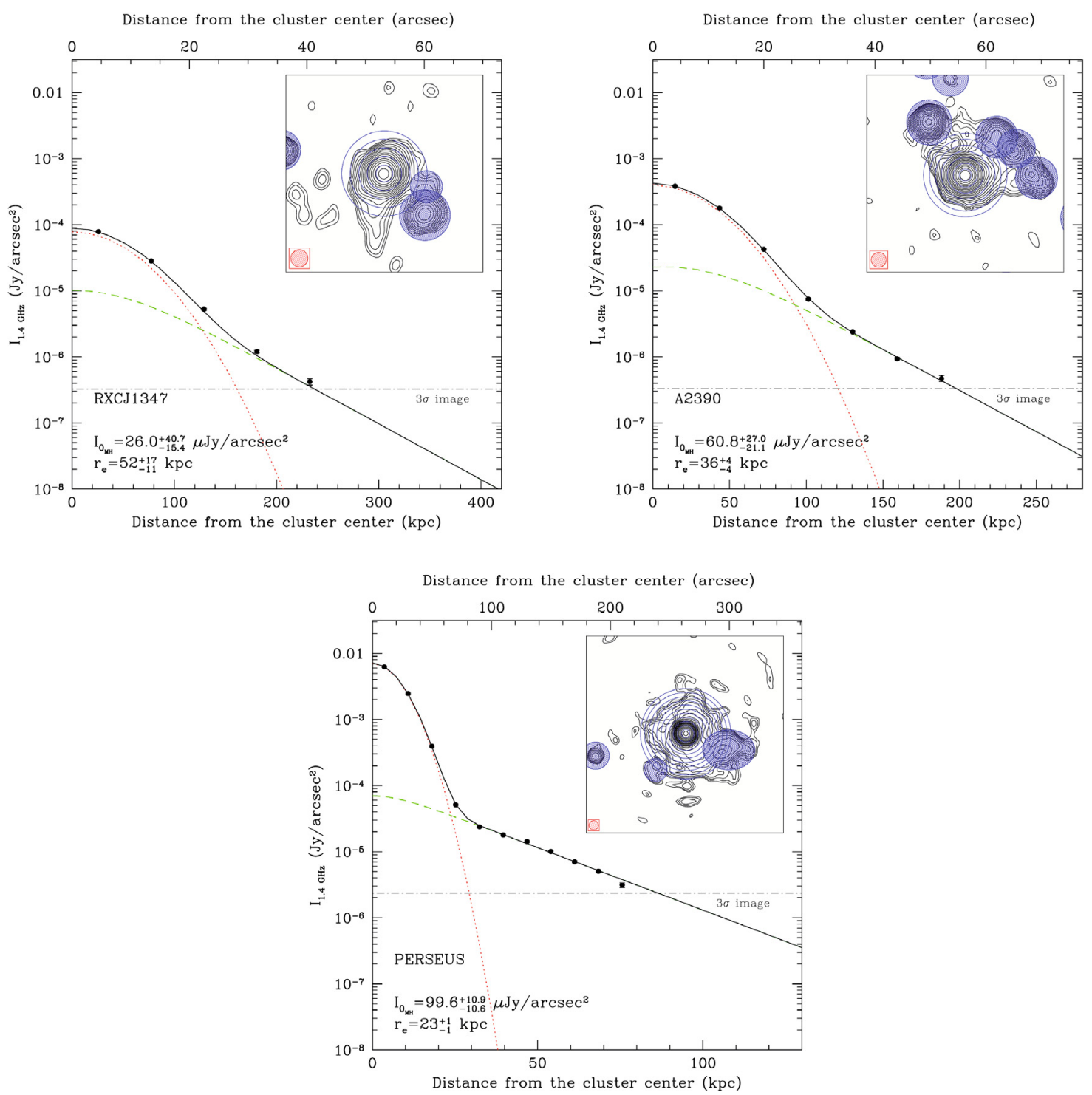

Fig. 4. Azimuthally averaged brightness profiles of the mini-halos in RXJ1347.5-1145, A2390, and Perseus, previously imaged in the literature (Gitti et al. 2007; Bacchi et al. 2003; Pedlar et al. 1990). The profiles have been calculated in concentric annuli, as shown in the inset panels. All the discrete sources (except the central one) have been masked. For each cluster the horizontal dashed-dotted line indicates the $3 \sigma$ noise level of the radio image. In the analysis we considered all the data points above the $3 \sigma$ radio noise level. The black line indicates the best fit profile described by an exponential law (dashed line), representing the mini-halo emission, and by a central Gaussian profile (dotted line), representing the central point source.

than $I_{0} \gtrsim 1 \mu \mathrm{Jy} / \operatorname{arcsec}^{2}$. As a result, the observed tendency for the smaller mini-halos to be brighter must be considered prudently. In any case, the possible selection effect mentioned above seems not to influence the result that mini-halos have systematically lower values of $r_{e}$ than halos. Mini-halos appear effectively smaller than halos and this could reflect the higher density of the intergalactic gas in the central regions of cooling clusters.

For a better comparison between halos and mini-halos in terms of emissivity, in Fig. 5 (bottom panel) we show the distribution of the radio emissivity (calculated by using Eq. (9)) both for halos (in blue) and mini-halos (in red). We find that radio halos have a surprisingly narrow radio emissivity distribution around an average value of about $10^{-42} \mathrm{erg} \mathrm{s}^{-1} \mathrm{~cm}^{-3} \mathrm{~Hz}^{-1}$. In contrast, mini-halos span a wide range of radio emissivity. Some of them, like the Perseus mini-halo, would be characterized by a radio emissivity more than 100 times larger than that of a typical halo, quite in agreement with the recent finding by Cassano et al. (2008). We note, however, that the three new mini-halos analyzed in this work are all characterized by brightness and size very similar to that of the smaller halos and thus their radio emissivity is comparable to that of halos in general.

As a further check, we also examined the trend of the radio power (calculated from the flux density in Eq. (6)) versus the $e$-folding radius of mini-halo and halos, see Fig. 6. This plot is similar to that presented in Cassano et al. (2008) except that they calculated the radius as $\sqrt{r_{\min } \times r_{\max }}, r_{\min }$ and $r_{\max }$ being the minimum and the maximum radius measured using the $3 \sigma$ radio isophotes. As pointed out by Cassano et al. (2007), the powerful radio halos are the more extended sources while the fainter ones are smaller in size. Figure 6 shows indeed a tight correlation between the radio power and the $e$-folding radius of radio halos. We find that the radio power increases as $P_{1.4} \propto$ $r_{e}^{3}$. In our analysis, the slope of the correlation is in agreement with the finding that the radio emissivity has a very small scatter among radio halos. In fact, the radio power scales as $P_{1.4}=J \cdot V$, where $V$ is the source volume and $J$ is the radio emissivity. If 

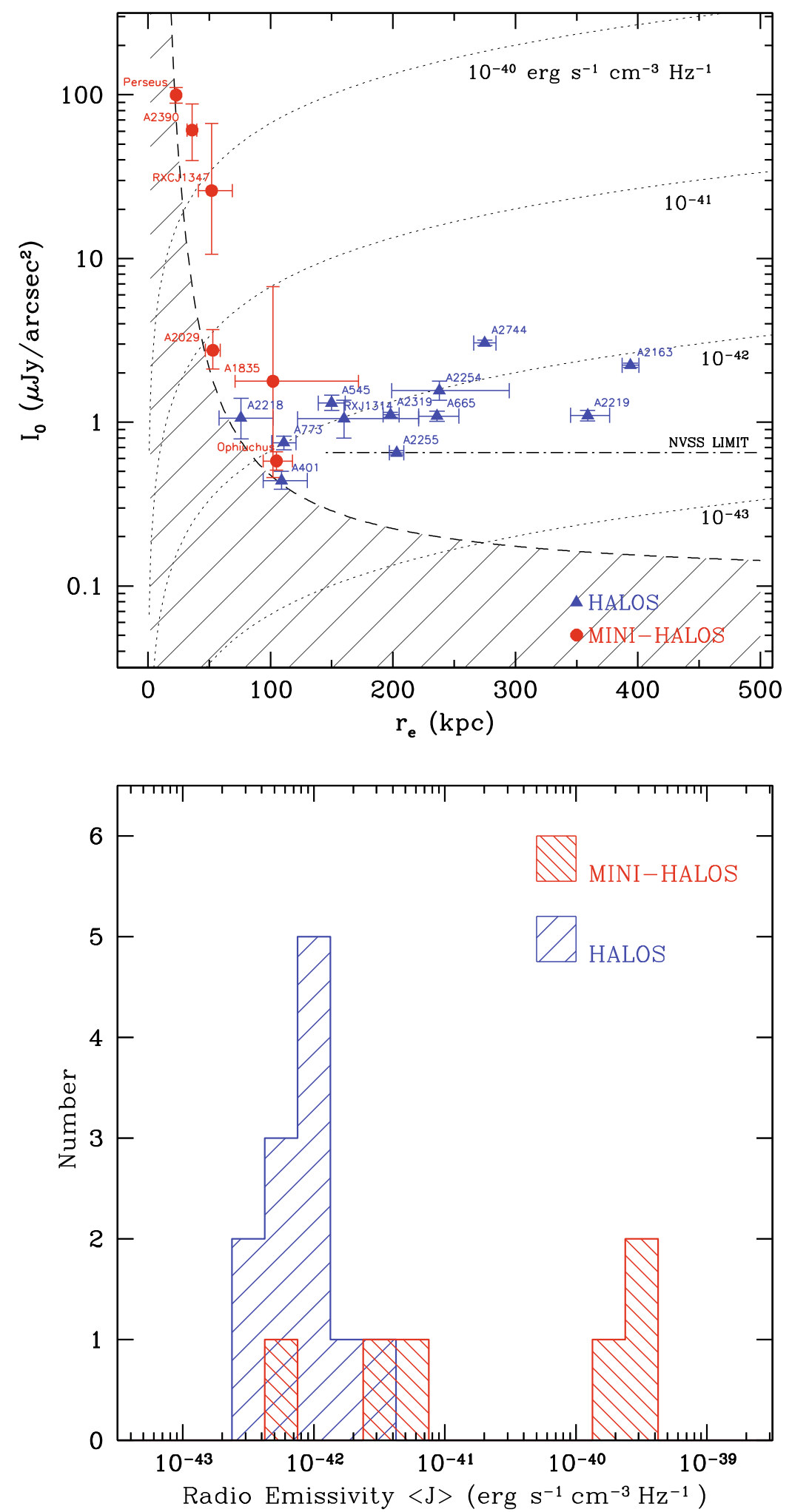

Fig. 5. Top: best fit central brightness $I_{0}\left(\mu \mathrm{Jy} / \operatorname{arcsec}^{2}\right.$ ) versus the length-scale $r_{e}$ (kpc) both for halos (blue triangles) and mini-halos (red dots). The dotted lines indicate regions of constant emissivity, namely $0.1,1,10,100$ times the average emissivity of radio halos which is $\langle J\rangle \simeq$ $10^{-42} \mathrm{erg} \mathrm{s}^{-1} \mathrm{~cm}^{-3} \mathrm{~Hz}^{-1}$. They have been traced from Eq. (9) by assuming a putative redshift of $z=0.18$ (the average redshift of our sample) and a spectral index $\alpha=1$. The dashed line represents the detection limit expected for a mini-halo or a halo at $z=0.18$ observed in a deep image with a beam of $25^{\prime \prime}$ and a sensitivity level of $25 \mu \mathrm{Jy} / \mathrm{beam}$, see text. The dot-dashed line represents the $3 \sigma$ sensitivity level of the NVSS. Bottom: Distribution of the $k$-corrected radio emissivities listed in Tables 2 and 3 both for halos (in blue) and mini-halos (in red).

we assume that the emissivity does not change from one halo to the other it follows that $P_{1.4} \propto r_{e}^{3}$. The dotted reference line in Fig. 6 represents the expected radio power if we assume for the emissivity the average value we found for radio halos $\langle J\rangle \simeq$ $10^{-42} \mathrm{erg} \mathrm{s}^{-1} \mathrm{~cm}^{-3} \mathrm{~Hz}^{-1}$ and for the volume $V$ the volume of a sphere a radius $3 r_{e}: \log P_{1.4} \simeq 23.52+3 \log \left(r_{e} / 100 \mathrm{kpc}\right)$. 


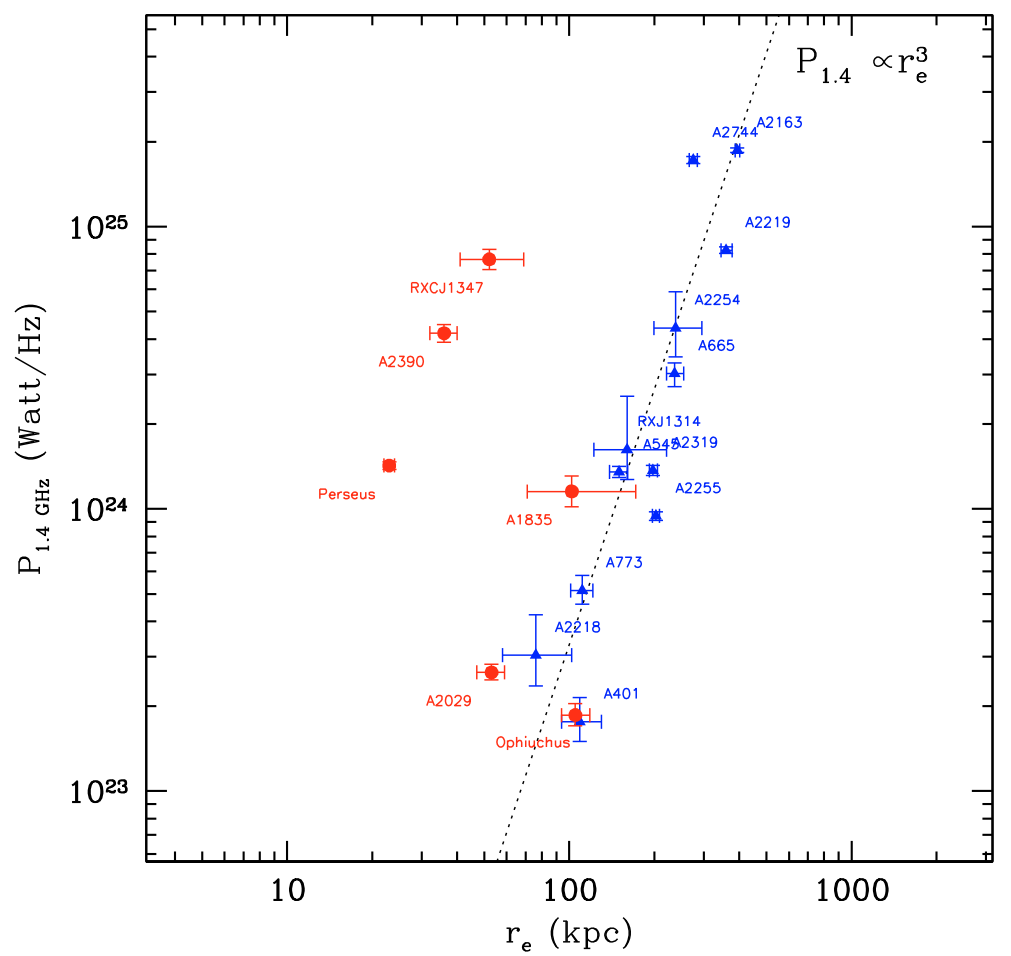

Fig. 6. Radio power at $1.4 \mathrm{GHz}$ versus $e$-folding radius in kilosparsecs for mini-halos (red dots) and halos (blue triangles). The dotted reference line represents the expected radio power if we assume for the emissivity the average value we found for radio halos $\langle J\rangle=10^{-42} \mathrm{erg} \mathrm{s}^{-1} \mathrm{~cm}^{-3} \mathrm{~Hz}^{-1}$ and for the volume $V$ the volume of a sphere a radius $3 r_{e}: \log P_{1.4} \simeq 23.52+3 \log \left(r_{e} / 100 \mathrm{kpc}\right)$.

We note that Cassano et al. (2008) found also a correlation between the power and the size of mini-halos. In fact, the three mini-halos we have in common (A2390, RXCJ1347, and Perseus) all seem to be aligned in a similar but offset correlation with respect to radio halos. However, with the addition of the new objects found in Paper I and analyzed here, we cannot define any clear correlation for the mini-halos, in agreement with the large spread in emissivity shown in Fig. 5. The mini-halos in A1835, Ophiuchus, and A2029 appear clearly separated from the other mini-halos although this separation into two distinct groups is likely just due to the small-number statistics.

As a final remark, we stress once again that our analysis should be relatively independent of the noise level of the radio images. To ensure that our approach is not dependent on the sensitivity of the radio observation we retrieved for A2744 (one of the clusters analyzed here) the NVSS image. We derived the azimuthally averaged brightness profile as computed for the deeper observations (same rings and mask), and we then fit the NVSS profile with an exponential law. The resulting fit is shown in the left panel of Fig. 7. The contours of the NVSS image are shown on the top right panel of Fig. 7 in comparison with the contours of the deeper VLA images on the bottom right panel. As expected, the radius as derived from the contour levels yields very different values in images with different sensitivities. One should consider that the same effect may appear in images with comparable sensitivity of objects characterized by the same intrinsic size but different brightness. Fainter halos (or mini-halos) tend to "sink" into the noise appearing smaller than they really are. On the other hand, in comparing the fitting results of the NVSS profiles in Fig. 7 with the fitting results of the deeper images in Table 3, we find the results to be in good agreement. Therefore we are confident that the method discussed here, based on the determination of length-scale and central brightness in extended structures, is less subject to the $\mathrm{S} / \mathrm{N}$ ratio of the radio images and thus better suited for comparative studies of the general properties of diffuse halo-like sources.

\section{Monte Carlo statistical analysis of the fitting procedure}

The fit of the exponential model described in Sect. 2 could be critical in the case of those mini-halos in which the $e$-folding radius is smaller than the $F W H M$ of the central point source. The mini-halos in A1835, A2029, RXCJ1347, and A2390 are all in this category. However, it should be noted that not only the observing resolution but also the brightness of the mini-halo is crucial for the determination of the $e$-folding radius. If the mini-halo is bright enough, the exponential profile will emerge from the central point source and it will be observable out to a large distance from the cluster center, making the fit of the $e$-folding radius $r_{e}$ possible. The example of A2029 is representative of this situation. The $e$-folding radius derived from the fit, $r_{e}=37^{\prime \prime} \pm 4^{\prime \prime}$, is below the FWHM beam of the observation which is $53^{\prime \prime}$. Nevertheless, the relative intensity of the minihalo with respect to the noise permits the fit of the exponential model from the external part of the mini-halo profile that does not overlap with the central point source. The limiting cases of A1835 and RXC J1347 (the two more distant mini-halos in our sample) are more critical and the behaviour of the fitting procedure must be carefully checked.

In order to assess the reliability of the best fit parameters, we created a set of Monte Carlo simulations with the aid of the Cybersar-OAC computer cluster. For each mini-halo we varied the values of $I_{0}$ and $r_{e}$ in a regular grid that contained the best fit parameters reported in Table 2 and 3 . For each pair of $I_{0}$ and $r_{e}$ values, we created a synthetic image with the same field of view, pixel size, FWHM beam, noise level, and masked regions as that observed. A central point source with the same intensity 

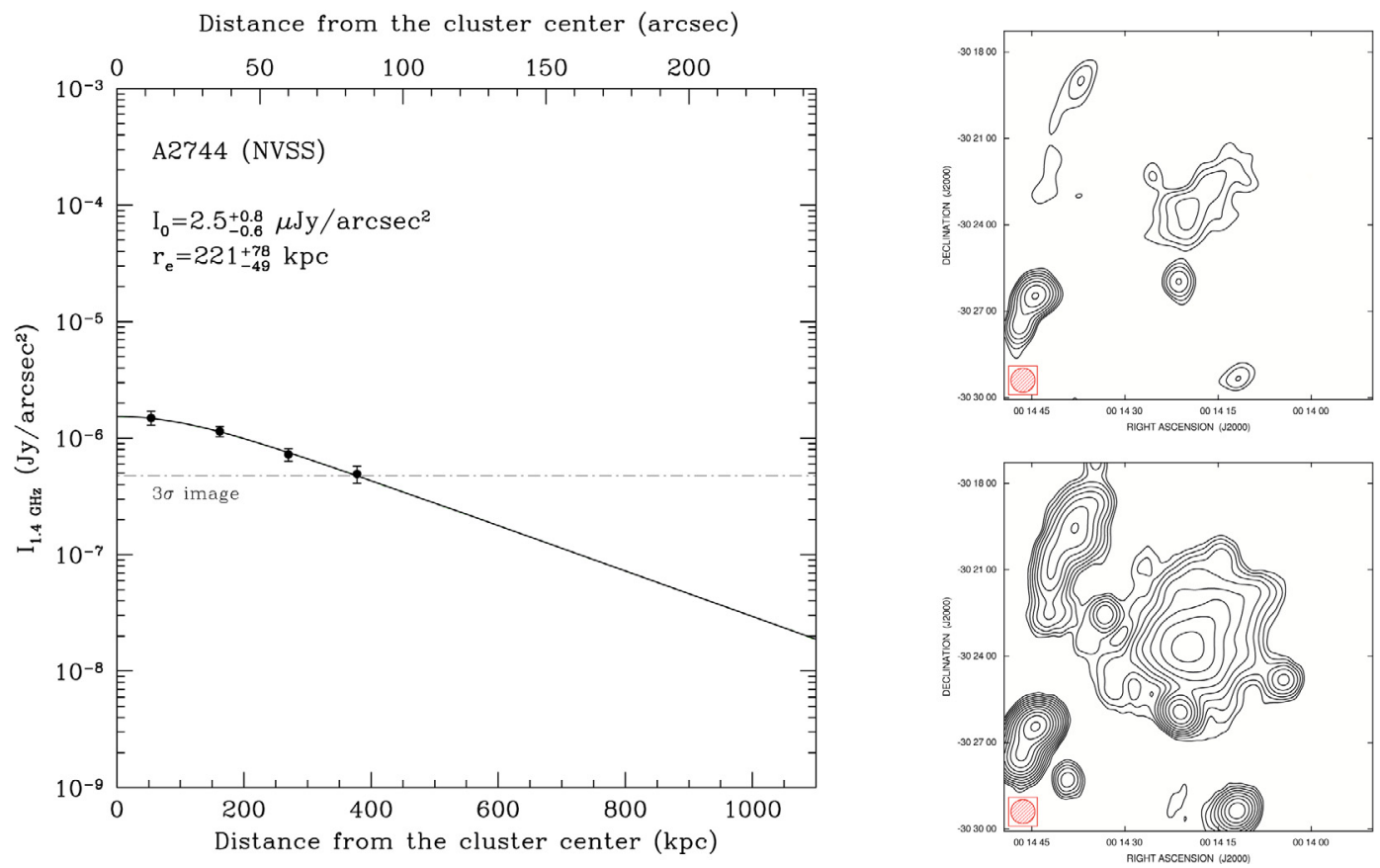

Fig. 7. Left: fit of the azimuthally averaged brightness profiles calculated from the NVSS image of the cluster A2744. Right: contours of the NVSS image in comparison with the contours of the deeper VLA image for A2744 (Govoni et al. 2001a). In both images the first contour is drawn at $3 \sigma$ and the rest are spaced by a factor $\sqrt{2}$. The sensitivity $(1 \sigma)$ of the NVSS is $0.45 \mathrm{mJy} / \mathrm{beam}$ while the sensitivity of the deeper image is $0.1 \mathrm{mJy} / \mathrm{beam}$. The radius as derived from the contour levels depends on the sensitivity of the radio image, while the fit procedure give similar results in images at different sensitivity.
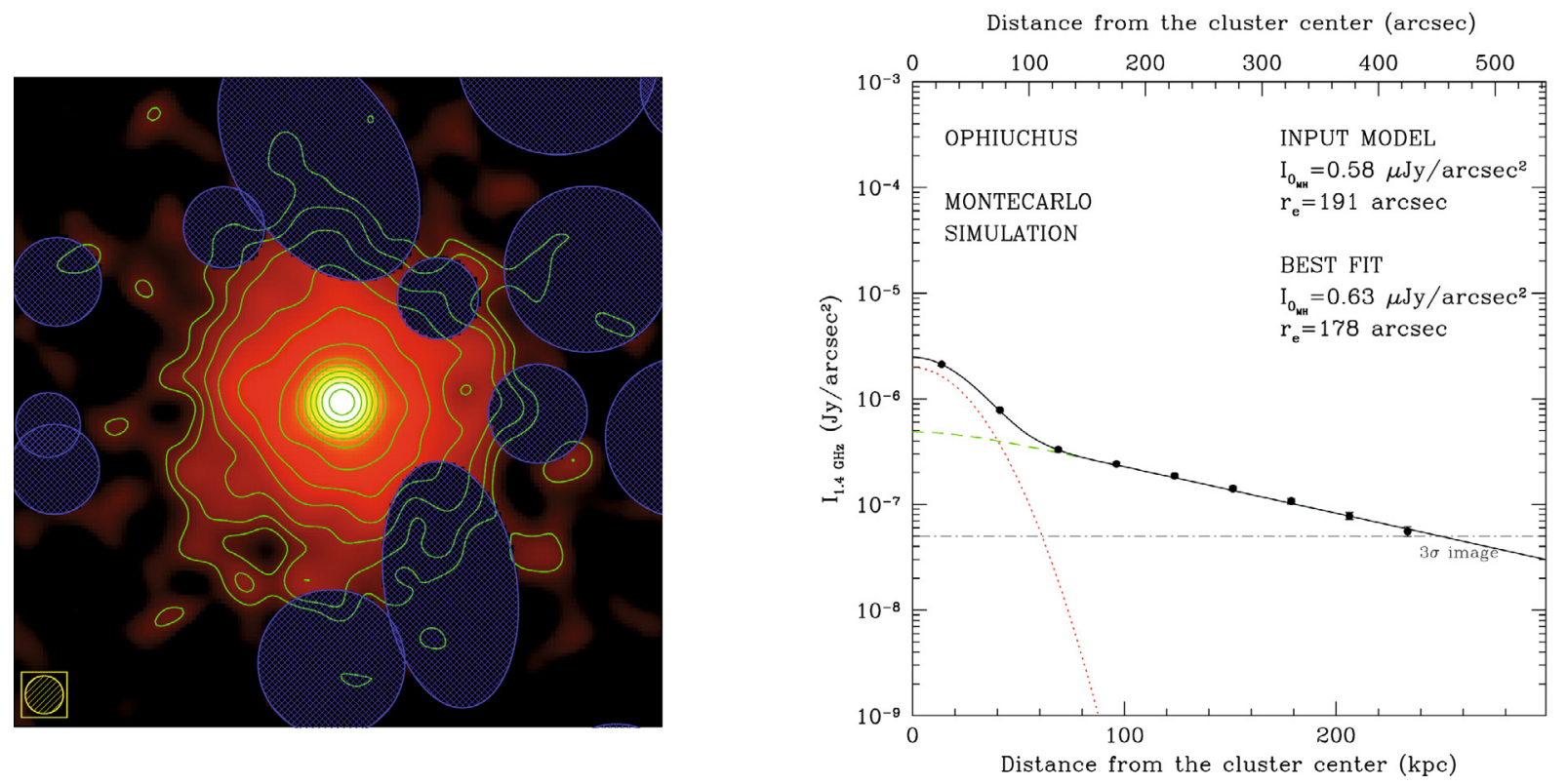

Fig. 8. Synthetic image (left panel) and fit profile (right panel) drawn from the Monte Carlo simulation of the Ophiuchus mini-halo.

as the observed one is added to the exponential model. An example of such image, drawn from the Monte Carlo simulation of the Ophiuchus mini-halo, is presented in left panel of Fig. 8. We then feed the synthetic image into the fit procedure and check for any systematic difference between the input and the output values of $I_{0}$ and $r_{e}$. In the right panel of Fig. 8 we show the fit obtained from the synthetic image on the left. We can see that in the case of the Ophiuchus mini-halo, which is particularly extended, the difference between input and the output values of $I_{0}$ and $r_{e}$ is fully consistent with the uncertainties reported in Table 2. In Fig. 9 we present the result of the Monte Carlo simulations for all six mini-halos analyzed in this work. For each mini-halo we plot the grid of the input values of $I_{0}$ and $r_{e}$ (crosses) with the relative arrows representing the displacement of the output parameters from the fit procedure. The dashed line represents the detection limit for each mini-halo and has been traced with the same criterion as in top panel of Fig. 5. Here the exact values for the $F W H M$ beam and noise level of the specific observations have been used. In each panel, the dot indicates the values of the actual best fit parameters with their uncertainties. Clearly, for bright and extended mini-halos, the fit procedure is able to recover the original parameters with high precisions. Obviously, 
M. Murgia et al.: Comparative analysis of the diffuse radio emission in the galaxy clusters A1835, A2029, and Ophiuchus
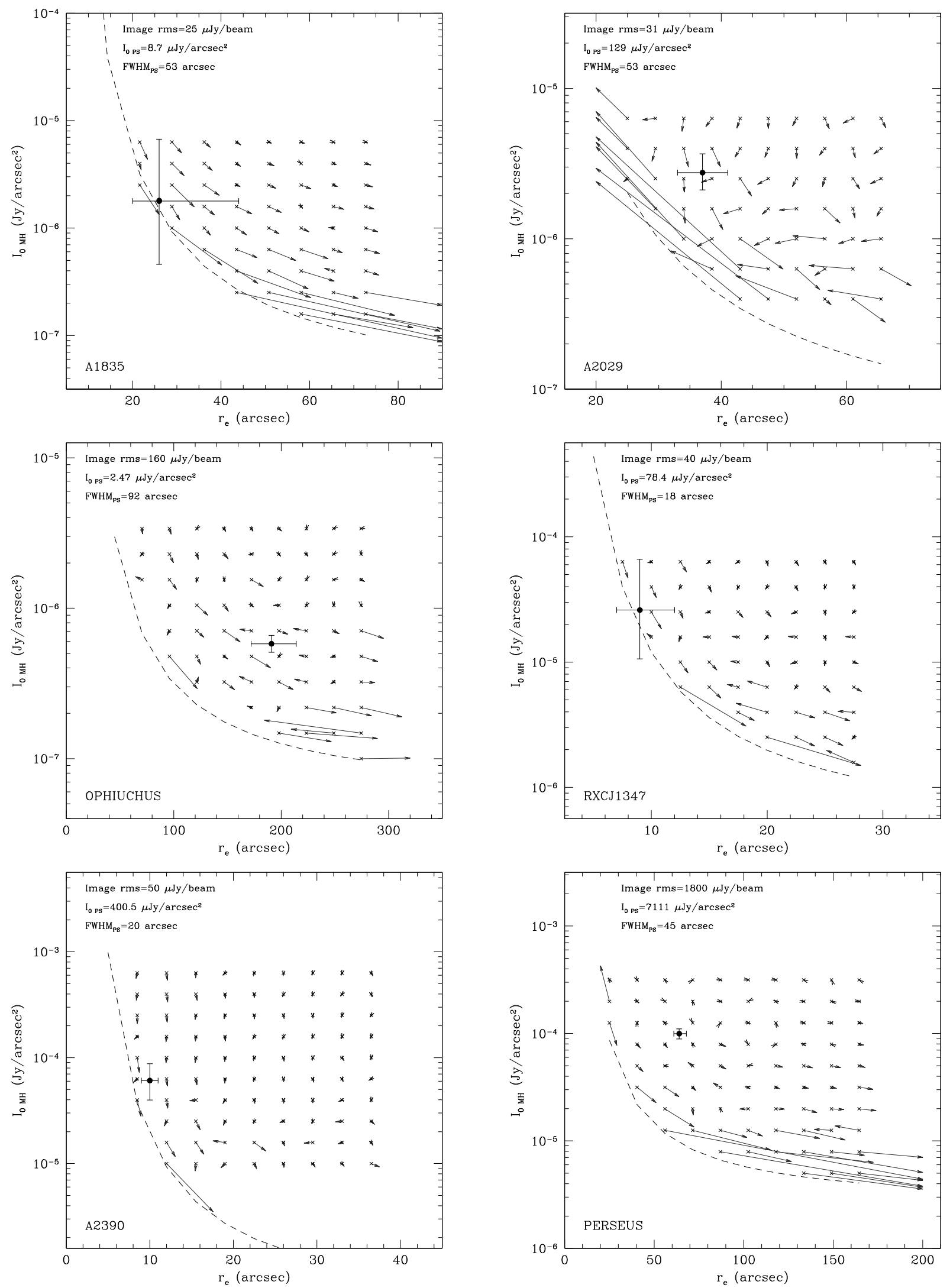

Fig. 9. Monte Carlo simulations for the six mini-halos analyzed in this work, see text for further details.

near the detection limit the situation can be much worse and large systematic effects are observed. However, we note that the fit procedure is able to recover the input $e$-folding even if it is significantly smaller then the $F W H M$ beam, provided that the value of $I_{0}$ is sufficiently high (see e.g. the case of A2390 with $r_{e} \simeq 10^{\prime \prime}$ and $I_{0}>10 \mu \mathrm{Jy} /$ beam).

In summary, the results of the Monte Carlo simulation tell us that the fit procedure is able to constraint the model parameters 
M. Murgia et al.: Comparative analysis of the diffuse radio emission in the galaxy clusters A1835, A2029, and Ophiuchus
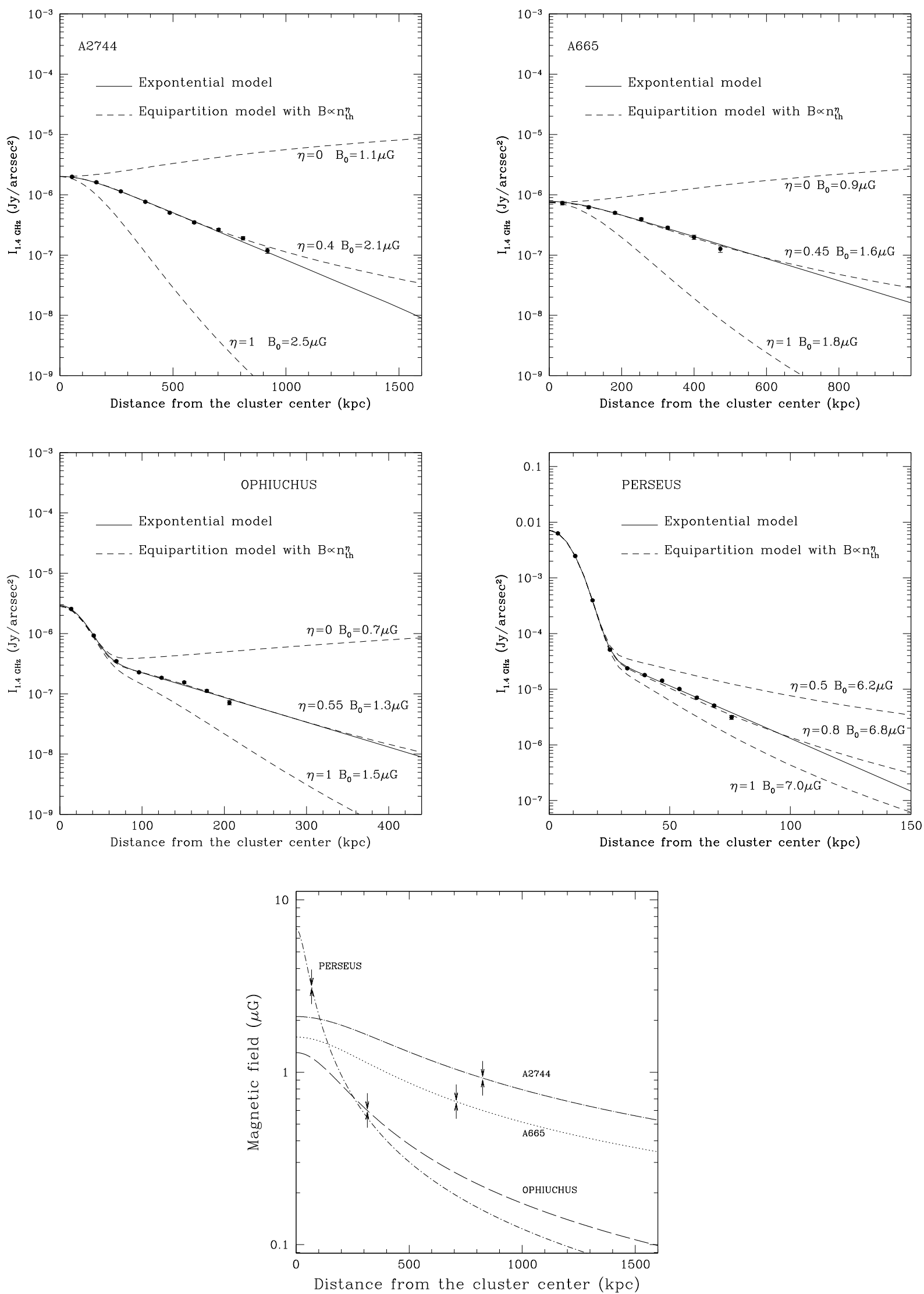

Fig. 10. Comparison of the exponential (solid line) and equipartition models (dashed lines) with $B \propto n_{\text {th }}^{\eta}$ for the radio halos in A2744 and A655 (top panels) and for the mini-halos in Ophiuchus and Perseus clusters (middle panels). The value of the central magnetic field strength, $B_{0}$, is reported along with the value of $\eta$ for each profile. Bottom panel shows the magnetic field trends for the best equipartition profiles. The arrows indicate the position of the $3 r_{e}$ obtained by the fit using the exponential model. 


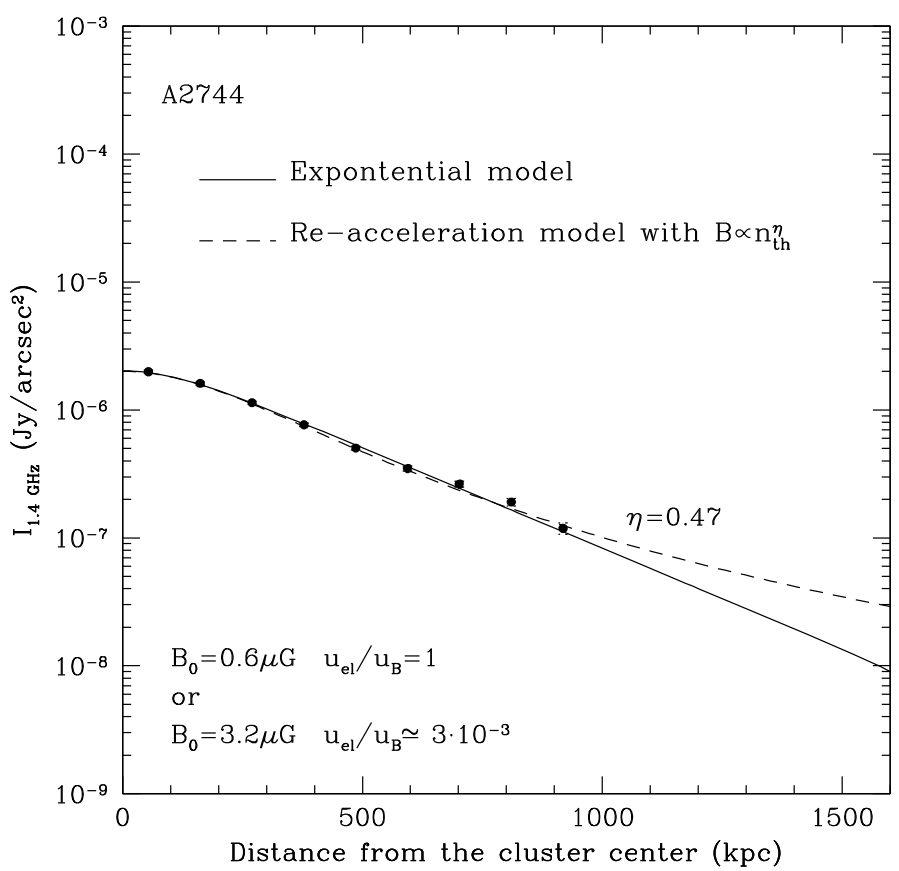

Fig. 11. Abell 2744. Comparison of the exponential (solid line) and reacceleration model (dashed line).

with accuracy and without systematic effects in the cases of Ophiuchus, Perseus, A2029, and A2390. The mini-halos in A1835 and RXCJ1347 are very close to the detection limit and the best fit parameters could be affected by a systematic bias. In particular, they could be smaller and brighter than what is inferred by the fit to the exponential model, although the bias appears to be comparable to the reported uncertainties.

\section{Discussion}

The exponential model fitting method provides an estimate of the brightness and size of the diffuse, halo-like, radio emission in cluster of galaxies using a minimal set of free parameters. In this section we discuss the exponential profile in comparison with alternative models which may have a much clearer physical meaning, but require the introduction of a number of additional assumptions and/or free parameters.

One main difficulty in the study of the non-thermal radio halos and mini-halos in clusters of galaxies is that the synchrotron emissivity essentially traces the product of electron and magnetic field energy densities so disentangling the two contributions is not easy. Another source of uncertainty is related to the effective shape of the energy spectrum of the relativistic electrons across the cluster which is poorly known. Given their comparatively short radiative life-times, the synchrotron electrons cannot diffuse into the large volume of space involved but rather they must be either continuously injected and/or reaccelerated in situ throughout the intra-cluster medium (e.g., Dennison 1980; Jaffe 1977; Dolag \& Ensslin 2000; Brunetti et. al 2001). The spectral energy distributions for the cosmic ray electrons predicted by these models can be quite different, however from the current data we are not able to discriminate between the two processes definitively. Our intent here, is to highlight the role of the magnetic field in determining the radial profile of the radio emission. For illustrative purposes, in the following we discuss simplified models for the distribution of particles and magnetic fields in clusters that are often discussed in literature and we compare them with the exponential profile.

\subsection{Injection models}

We can compare a continuous injection of cosmic ray electrons in the intra-cluster medium with a power law distribution in the energy range from $\gamma_{\min } m_{e} c^{2}$ to $\gamma_{\max } m_{e} c^{2}\left(\gamma_{\max } \gg \gamma_{\min }\right)$. In the stationary case, and for an emission spectrum with index $\alpha=1$, from standard synchrotron formulas (Rybicki \& Lightman 1979) we obtain for the radio emissivity:

$J_{v}=2.21 \times 10^{-10} \frac{Q_{\mathrm{el}}}{B_{\mu \mathrm{G}}^{2}+B_{\mathrm{CMB}}^{2}} B_{\mu \mathrm{G}}^{2} v_{\mathrm{GHz}}^{-1}\left(\mathrm{erg} \mathrm{s}^{-1} \mathrm{~cm}^{-3} \mathrm{~Hz}^{-1}\right)(10)$

where $B_{\mathrm{CMB}}=3.25(1+z)^{2} \mu \mathrm{G}$ is the equivalent magnetic field associated with the cosmic microwave background while $Q_{\mathrm{el}}$ is the production rate of relativistic electrons in units of erg s$~^{-1} \mathrm{~cm}^{-3}$. In Eq. (10) we supposed that the electron population is isotropic and we average over all the possible directions between the magnetic field and the line-of-sight, i.e. the field is completely tangled.

The energy density of the cosmic ray electrons can be calculated as

$u_{\mathrm{el}} \simeq 7.74 \times 10^{20} \frac{Q_{\mathrm{el}}}{B_{\mu \mathrm{G}}^{2}+B_{\mathrm{CMB}}^{2}} \gamma_{\mathrm{min}}^{-1} \quad\left(\mathrm{erg} \mathrm{cm}^{-3}\right)$.

In the case of a perfect equipartition between the energy densities of particles and fields $\left(u_{\mathrm{el}}=B^{2} / 8 \pi\right)$, we have that the radio source is near minimum energy conditions and the radio emissivity at $1.4 \mathrm{GHz}$ is given by:

$J_{1.4} \simeq 8.12 \times 10^{-43} \gamma_{\min 100} B_{\mu \mathrm{G}}^{4}\left(\mathrm{erg} \mathrm{s}^{-1} \mathrm{~cm}^{-3} \mathrm{~Hz}^{-1}\right)$

The synchrotron emissivity is indeed very close to the observed value of $\langle J\rangle \simeq 10^{-42} \mathrm{erg} \mathrm{s}^{-1} \mathrm{~cm}^{-3} \mathrm{~Hz}^{-1}$ in the case of radio halos for a low energy cut-off of $\gamma_{\min }=100$ and an average magnetic field strength of about $1 \mu \mathrm{G}$ (changing $\gamma_{\text {min }}$ from 10 to 1000 causes $B$ to vary from 1.8 to $0.5 \mu \mathrm{G}$ ). Assuming a redshift of $z=0.18$ (the average distance of the radio halos in our sample), from Eq. (11) we find that the required energy supply to explain the observed radio halos emissivity in a minimum energy condition is $Q_{\mathrm{el}} \simeq 1.1 \times 10^{-31} \mathrm{erg} \mathrm{s}^{-1} \mathrm{~cm}^{-3}$. Note that this power is about an order of magnitude larger than the total synchrotron luminosity and one order of magnitude less than the thermal X-ray luminosity.

The equipartition magnetic field strength quoted above should be regarded as volume averages. However, there are observational and theoretical arguments to support the idea that inside a cluster the magnetic field strength scales as a function of the thermal gas density, $n_{\text {th }}$, so:

$B(R)=B_{0}\left[\frac{n_{\mathrm{th}}(R)}{n_{\mathrm{th}}(0)}\right]^{\eta}$

where the index $\eta$ is expected to be in the range $0.3-1$ (e.g., Dolag et al. 2001, 2002; Murgia et al. 2004; Guidetti et al. 2008; Battaglia et al. 2009).

If the density profile of the thermal gas is described by the standard $\beta$-model (Cavaliere \& Fusco-Femiano 1976), from Eq. (12) we have

$J_{1.4}(R)=J_{0}\left(1+R^{2} / r_{\mathrm{c}}^{2}\right)^{-6 \beta \eta}$ 
where $r_{\mathrm{c}}$ is the cluster's core radius. The radio brightness profile is:

$I_{1.4}(r)=I_{0}\left(1+r^{2} / r_{\mathrm{c}}^{2}\right)^{-6 \beta \eta+0.5}$

where $r$ is the projected distance from the cluster center. It is worthwhile to note that the ratio between the radio and X-ray brightness is proportional to

$\frac{I_{1.4}}{I_{X}} \propto T^{-1 / 2}\left(1+r^{2} / r_{\mathrm{c}}^{2}\right)^{-3 \beta(2 \eta-1)}$

where $T$ is the gas temperature. Thus, if $\eta=0.5$, a linear relation is expected between the radio and the X-ray brightness for a perfectly isothermal $\beta$-model.

We now reconsider the radio halos in A2744 and A665, for which an average spectral index $\alpha \simeq 1$ has been determined from VLA radio images at $327 \mathrm{MHz}$ and $1.4 \mathrm{GHz}$ by Orrù et al. (2007) and Feretti et al. (2004), respectively. In the top panels of Fig. 10, we trace the equipartition model profiles by converting to our cosmology the $\beta$-model parameters reported by Kempner \& David (2004) and Birkinshaw et al. (1991) for $\mathrm{A} 2744\left(r_{\mathrm{c}}=382 \mathrm{kpc}\right.$ and $\left.\beta=0.79\right)$ and A665 $\left(r_{\mathrm{c}}=291 \mathrm{kpc}\right.$ and $\beta=0.66)$, respectively. The equipartition model is very sensitive to the radial profile of the magnetic field strength. In order to show this behaviour, we fix $I_{0}$ in Eq. (15) and choose three particular values for $\eta$. A constant field, $\eta=0$, results in a profile which is too flat with respect to the data while a magnetic field whose strength decreases with the distance as the gas density, $\eta=1$, results in a profile which is too steep. A very good agreement with the data is found for $\eta \simeq 0.4-0.5$ for both radio halos, implying a magnetic fields whose energy density is proportional to that of the thermal gas. Indeed, in A2744 a linear correlation between the radio and X-ray surface brightness has been observed (Govoni et al. 2001b). Moreover, a comparison of the equipartition model with $\eta=0.5$ and the exponential fit shows that the two profiles are strikingly similar within one megaparsec from the cluster center. The differences between the two profiles become appreciable only at much larger distances, where the radio halo emission has fallen below the noise level.

In the bottom panels of Fig. 10 we show the exponential and equipartition profiles for the mini-halos in the Ophiuchus and Perseus clusters. We adopt a core radius of $r_{\mathrm{c}}=199 \mathrm{kpc}$ and $\beta=0.747$ for Ophiuchus, and $r_{\mathrm{c}}=44 \mathrm{kpc}$ and $\beta=0.54$ for Perseus (Chen et al. 2007). We note that the equipartition profile in Eq. (15) should be considered only outside the cool core where the $\beta$-model still provides a reasonable description of the gas density. Moreover, Ophiuchus lacks any spectral index information while the mini-halo in Perseus has an average spectral index which is slightly steeper than what we have assumed here ( $\alpha \simeq 1.2$, Gitti et al. 2004). Apart from these caveats, however, the exponential and the equipartition profiles are remarkably similar in both mini-halos. As found for the radio halos in A2744 and A665, the mini-halo in Ophiuchus is well described by an equipartition model with $\eta \simeq 0.5$. In the case of the Perseus mini-halo, however, we found $\eta \simeq 0.8$, which implies a particularly steep magnetic field radial profile (this result does not change significantly by assuming $\alpha=1.2$ instead of $\alpha=1$ ). Indeed, Burns et al. (1992) suggested that the absence of a cluster-wide magnetic field inhibits a large scale halo in Perseus.

We conclude that the exponential profile is very close to the expectations of a simple equipartition model if the magnetic field energy density roughly scales as the thermal gas density. In the framework of the equipartition model, the physical meaning of the $3 r_{e}$ length-scale obtained by the exponential fit is that it marks the point at which the magnetic field strength is decreased to about half the value at the cluster center (see bottom panel of Fig. 10). Hence, mini-halos would appear smaller because the magnetic field falls off more rapidly with radius.

It is worthwhile to note that if $B<B_{\mathrm{CMB}}$ and $\eta \simeq 0.5$ (as for A2744, A665 and Ophiuchus), the equipartition condition implies that the injection rate should scale roughly as the thermal gas density $Q_{\mathrm{el}} \propto n_{\text {th }}$. In models that consider a continuous production of secondary electrons by hadronic collisions in the intra-cluster medium (Dennison 1980), the injection rate of cosmic ray electrons is proportional to the product of the densities of the gas and cosmic ray protons, $Q_{\mathrm{el}} \propto n_{\mathrm{th}} n_{\mathrm{CRp}}$. Usually, it is assumed that $n_{\mathrm{CRp}} \propto n_{\mathrm{th}}$ and as a results $Q_{\mathrm{el}} \propto n_{\mathrm{th}}^{2}$. As a consequence secondary models predict brightness profiles that are generally too steep to explain observed trends in radio halos. However, the radio brightness profiles in the Perseus mini-halo is steeper and it can be described by secondary models as well (Pfrommer \& Ensslin 2004).

\subsection{Re-acceleration models}

The observed radio profiles may be also described in the framework of the re-acceleration scenario (e.g. Gitti et al. 2004). An over-simplified re-acceleration model may be considered in which the relativistic electrons energy spectrum is essentially peaked at a characteristic energy, $\gamma_{\max } m c^{2}$, at which the reacceleration gains balance the radiative losses. In the stationary case, the energy of the cosmic ray electrons can be calculated as:

$u_{\mathrm{el}} \simeq 7.74 \times 10^{20} \frac{Q_{\mathrm{el}}}{B_{\mu \mathrm{G}}^{2}+B_{\mathrm{CMB}}^{2}} \gamma_{\max }^{-1} \quad\left(\mathrm{erg} \mathrm{cm}^{-3}\right)$

where $Q_{\mathrm{el}}=\dot{u}_{\mathrm{el}}$ is the energy supply to the re-acceleration process in units of erg s $\mathrm{s}^{-1} \mathrm{~cm}^{-3}$.

The emission spectrum will extend up to a maximum frequency of $v_{\max }=4.2 \times 10^{-9} B_{\perp \mu \mathrm{G}} \gamma_{\max }^{2} \mathrm{GHz}$, beyond which it cuts off exponentially. The synchrotron emissivity in cgs units is:

$J_{v}=2.74 \times 10^{-10} \frac{Q_{\mathrm{el}}}{B_{\mu \mathrm{G}}^{2}+B_{\mathrm{CMB}}^{2}} B_{\mu \mathrm{G}}^{2} v_{\max }^{-1} F\left(v / v_{\max }\right)$

where $F\left(v / v_{\max }\right)$ is the usual synchrotron kernel (Pacholczky 1970) while $v$ and $v_{\max }$ are expressed in GHz.

In order to reproduce a spectral index $\alpha_{325 \mathrm{MHz}}^{1.4 \mathrm{GHz}}=1$ between $325 \mathrm{MHz}$ and $1.4 \mathrm{GHz}$, it should be $v_{\max } \simeq 0.56 \cdot(1+z) \mathrm{GHz}$. At a frequency of $v=1.4 \mathrm{GHz}$ and at $z=0.18$, the average redshift of the sources in our sample, we can calculate $F\left(v / v_{\max }\right) \simeq 0.27$ (a steeper spectrum with $\alpha_{325 \mathrm{MHz}}^{1.4 \mathrm{GHz}}=2$ would imply $v_{\max } \simeq 0.31$. $(1+z) \mathrm{GHz}$ and $\left.F\left(v / v_{\max }\right) \simeq 0.06\right)$.

In the case of a perfect equipartition between the energy densities of particles and fields, $u_{\mathrm{el}}=B^{2} / 8 \pi$, we find that the radio source is near the minimum energy condition and the radio emissivity at $1.4 \mathrm{GHz}$ is:

$J_{1.4} \simeq 7.65 \times 10^{-41} B_{\mu \mathrm{G}}^{3.5} \quad\left(\mathrm{erg} \mathrm{s}^{-1} \mathrm{~cm}^{-3} \mathrm{~Hz}^{-1}\right)$.

The observed synchrotron emissivity in the case of radio halos, $\langle J\rangle \simeq 10^{-42} \mathrm{erg} \mathrm{s}^{-1} \mathrm{~cm}^{-3} \mathrm{~Hz}^{-1}$, implies an average magnetic field strength of about $0.3 \mu \mathrm{G}$. At a redshift $z=0.18$, from Eq. (18) it follows that the required energy supply is $Q_{\mathrm{el}} \simeq 2.2 \times 10^{-30} \mathrm{erg} \mathrm{s}^{-1} \mathrm{~cm}^{-3}$, about one order of magnitude larger than the required power found in the previous section in the case of the continuous injection model. 
A general result from Eq. (17) is that there exists a critical value for the magnetic field strength that minimize the acceleration efficiency $\chi=Q_{\mathrm{el}} / u_{\mathrm{el}}$ needed to radiate at a given $v_{\max }$, described by $B=B_{\mathrm{CMB}} / \sqrt{3}$. In the particular case of the average redshift of halos in our sample, the field that minimizes the re-acceleration efficiency results in $B \simeq 2.6 \mu \mathrm{G}$. Given this value for the magnetic field, from Eq. 18 it follows that the required energy supply to the re-acceleration process is $Q_{\mathrm{el}} \simeq 3.6 \times 10^{-32} \mathrm{erg} \mathrm{s}^{-1} \mathrm{~cm}^{-3}$, i.e. significantly less than in the equipartition case above. However, the energy density of cosmic ray electrons, $u_{\mathrm{el}} \simeq 1.3 \times 10^{-16} \mathrm{erg} \mathrm{cm}^{-3}$, is much lower than that of the field, $u_{B} \simeq 2.7 \times 10^{-13} \mathrm{erg} \mathrm{cm}^{-3}$, and thus the radio source cannot be in a condition of minimum energy.

In Fig. 11 we show the comparison of the re-acceleration model versus the exponential fit for the radio halo in A2744. The average spectral index of this radio halo is fairly constant with $\alpha_{325 \mathrm{GHz}}^{1.4 \mathrm{GHz}} \simeq 1$ (Orrù et al. 2007) which justifies the use of the same $v_{\max }$ at different distances from the cluster centre. By assuming that $B \propto n_{\text {th }}^{\eta}$, from Eq. (19) it follows that the radio brightness profiles is:

$I_{1.4}(r)=I_{0}\left(1+r^{2} / r_{\mathrm{c}}^{2}\right)^{-5.25 \beta \eta+0.5}$.

If the equipartition energy condition $u_{\mathrm{el}}=u_{B}$ is met at any radius, the re-acceleration model yields a central magnetic field of $B_{0} \simeq 0.6 \mu \mathrm{G}$ with a scaling index of $\eta=0.47$. If we fix $B_{0}=3.2 \mu \mathrm{G}$, we minimize the re-acceleration efficiency at the cluster centre but the radio halo is out of equipartition since the total energy density is dominated by the magnetic field energy density $\left(u_{\mathrm{el}} \simeq 3 \times 10^{-3} u_{B}\right)$. Whatever the case, we find that the exponential profile is also very close to the expectations of the simple re-acceleration model considered here.

\section{Conclusions}

Mini-halos in clusters are still poorly understood sources. They are a rare phenomenon, having been found so far only in few clusters. A larger number of mini-halos and better information on their physical properties will be necessary in order to discriminate between the different mechanisms suggested for transferring energy to the relativistic electrons that power the radio emission.

We recently found that at the center of the clusters A1835, A2029, and Ophiuchus, the dominant radio galaxy is surrounded by a diffuse low surface brightness mini-halo. We have studied the morphological and physical properties (i.e. length-scale, central brightness, emissivity) of these mini-halos by fitting their azimuthally averaged radio brightness profile with an exponential. The method proposed here to derive the length-scale of halos and mini-halos seems promising because it is relatively independent of the sensitivity of the radio observations. The exponential model is attractive in its simplicity and involves a minimal set of free parameters. While it cannot account for any local deviations from circular symmetry of the diffuse emission, this empirical method does provide a rough estimate of brightness and size of these sources. We compare the surface brightness profiles of the new mini-halos discovered in Paper I with data already available in the literature, both for mini-halos and halos. We find that the radio halos can have quite different length-scales but their emissivity is remarkably similar from one halo to another. This result could have important implications for theories of the origin of radio halos in clusters of galaxies but awaits confirmation from future, more sensitive, observations. In fact, if many faint halos have been missed by the current surveys, the average radio emissivity we found at $1.4 \mathrm{GHz},\langle J\rangle \simeq 10^{-42} \mathrm{erg} \mathrm{s}^{-1} \mathrm{~cm}^{-3} \mathrm{~Hz}^{-1}$, should be considered an upper limit. In contrast, mini-halos span a wide range of radio emissivity. Some of them, like the Perseus mini-halos, are characterized by a radio emissivity which is more than 100 times greater than that of radio halos. On the other hand, the new mini-halos in cooling core clusters analyzed here, namely A2029, Ophiuchus, and A1835, have a radio emissivity which is much more typical of halos in merging clusters rather than to the mini-halos previously known.

We discussed the exponential fit in comparison with the current theoretical models for the distributions of synchrotron electrons and magnetic fields in cluster of galaxies. We find that the exponential profile is very close to the expectations of these models if the magnetic field energy density roughly scales as the thermal gas density. In the framework of the equipartition model, the physical meaning of the $3 r_{e}$ length-scale obtained by the exponential fit is that it marks the point at which the magnetic field strength is decreased to about half the value at the cluster center. Mini-halos would appear smaller because the magnetic field falls off more rapidly with radius in cooling core clusters, in agreement with the suggestions of Burns et al. (1992).

Can we explain the larger dispersion in the emissivity of the mini-halos? We have very bright mini-halos, like Perseus, which host a particularly bright compact radio source at their center. For these, we cannot rule out that part of the mini-halo radio emission is related to properties of the local intergalactic medium and part is correlated with the AGN activity of the central brightest galaxy, as suggested by the faint correlation, discussed in Paper I, between the mini-halo and $\mathrm{cD}$ radio power. Indeed, the higher emissivity found in some mini-halos could be due to an extra-amount of energy supply from the strong AGN.

On the other hand, we also observe low-surface brightness mini-halos, like Ophiuchus, which host a faint AGN at their center. These mini-halos appear to be scaled down versions of the larger halos and they could be powered by similar merging processes. We note that, although cooling core clusters are generally considered relaxed system, when analyzed in detail they sometimes reveal peculiar X-ray features in the cluster center which may indicate a possible link between the mini-halo emission and some minor merger activity. Indeed, Burns et al. (2008) simulated the formation of both cool core and non-cool core clusters in the same numerical volume. These simulations confirmed that non-cool clusters are formed via major mergers early in their history which destroyed the cool cores and left significant residual kinetic energy in the gas which might be used to power the radio halos. In contrast, cool core clusters do not suffer any major mergers, thus preserving the central cool regions. However, the cool core clusters do experience regular smaller mergers which still inject energy in the intra-cluster medium, but more modestly than in the non-cool core clusters. These minor mergers might power the mini-halos.

Acknowledgements. We are grateful to an anonymous referee for very useful comments that improved the paper. This work is part of the "Cybersar" Project, which is managed by the COSMOLAB Regional Consortium with the financial support of the Italian Ministry of University and Research (MUR), in the context of the "Piano Operativo Nazionale Ricerca Scientifica, Sviluppo Tecnologico, Alta Formazione (PON 2000-2006)". F.G. and M.M. thank the hospitality of the Harvard-Smithsonian Center for Astrophysics where most of this research was done. Support was provided by Chandra grants GO5-6123X and GO6-7126X, NASA contract NAS8-39073, and the Smithsonian Institution. This research was partially supported by ASI-INAF I/088/06/0 - High Energy Astrophysics and PRIN-INAF2005. We wish to thank Pasquale Mazzotta for his valuable comments on the original draft. We are also grateful to Rossella Cassano and Chiara Ferrari for very useful discussions. The National Radio Astronomy Observatory 
(NRAO) is a facility of the National Science Foundation, operated under cooperative agreement by Associated Universities, Inc. This research has made use of the NASA/IPAC Extragalactic Data Base (NED) which is operated by the JPL, California Institute of Technology, under contract with the National Aeronautics and Space Administration.

\section{References}

Bacchi, M., Feretti, L., Giovannini, G., \& Govoni, F. 2003, A\&A, 400, 465 Birkinshaw, M., Hughes, J. P., \& Arnaud, K. A. 1991, ApJ, 379, 466

Battaglia, N., Pfrommer, C., Sievers, J. L., Bond, J. R., \& Enßlin, T. A. 2009, MNRAS, 393, 1073

Brunetti, G., Setti, G., Feretti, L., \& Giovannini, G. 2001, MNRAS, 320, 365

Buote, D. A. 2001, ApJ, 553, L15

Burns, J. O., Sulkanen, M. E., Gisler, G. R., \& Perley, R. A. 1992, ApJ, 388, L49

Burns, J. O., Hallman, E. J., Gantner, B., et al. 2008, ApJ, 675, 1125

Cassano, R., Brunetti, G., Setti, G., et al. 2007, MNRAS, 378, 1565

Cassano, R., Gitti, M., \& Brunetti, G. 2008, A\&A, 486, L31

Cavaliere, A., \& Fusco-Femiano, R. 1976, A\&A, 49, 137

Chen, Y., Reiprich, T. H., Böhringer, H., et al. 2007, A\&A, 466, 805

Condon, J. J., Cotton, W. D., Greisen, E. W., et al., 1998, AJ, 115, 1693

Dolag, K., \& Enßlin, T. A. 2000, A\&A, 362, 151

Dolag, K., Schindler, S., Govoni, F., \& Feretti, L. 2001, A\&A, 378, 777

Dolag, K., Bartelmann, M., \&Bottom Lesch, H. 2002, A\&A, 387, 383

Dunn, R. H., Fabian, A. C., \& Taylor, G. B. 2005, MNRAS, 364, 1343

Feretti, L., \& Giovannini, G. 2008, A Pan-Chromatic View of Clusters of Galaxies and the Large-Scale Structure, Lect. Notes Phys., 740, 143

Feretti, L., Giovannini, G., \& Böhringer, H. 1997, New Astron., 2, 501
Feretti, L., Fusco-Femiano, R., Giovannini, G., \& Govoni, F. 2001, A\&A, 373, 106

Bottom Feretti, L., Orrù, E., Brunetti, et al. 2004, A\&A, 423, 111

Feretti, L., Schuecker, P., Böhringer, H., Govoni, F., \& Giovannini, G. 2005, A\&A, 444, 157

Ferrari, C., Govoni, F., Schindler, S., et al. 2008, Space Sci. Rev., 134, 93

Giovannini, G., Tordi, M., \& Feretti, L. 1999, New Astron., 4, 141

Giovannini, G., \& Feretti, L. 2000, New Astron., 5, 335

Gitti, M., Brunetti, G., Feretti, L., \& Setti, G. 2004, A\&A, 417, 1

Gitti, M., Ferrari, C., Domainko, W., et al. 2007, A\&A, 470, L25

Govoni, F., Feretti, L., Giovannini, G., et al. 2001a, A\&A, 376, 803

Govoni, F., Enßlin, T. A., Feretti, L., \& Giovannini, G. 2001b, A\&A, 369, 441

Govoni, F., Markevitch, M., Vikhlinin, A., et al. 2004, ApJ, 605, 695

Govoni, F., Murgia, M., Feretti, L., et al. 2005, A\&A, 430, L5

Govoni, F., Murgia, M., Feretti, L., et al. 2006, A\&A, 460, 425

Govoni, F., Murgia M., Markevitch, M., et al. 2009, A\&A, 499, 371

Guidetti, D., Murgia, M., Govoni, et al. 2008, A\&A, 483, 699

Kempner, J. C., \& Sarazin, C. L. 2001, ApJ, 548, 639

Kempner, J. C., \& David, L. P. 2004, MNRAS, 349, 385

Murgia, M., Govoni, F., Feretti, L., et al. 2004, A\&A, 424, 429

Orrù, E., Murgia, M., Feretti, L., et al. 2007, A\&A, 467, 943

Pedlar, A., Ghataure, H. S., Davies, R. D., et al. 1990, MNRAS, 246, 477

Petrosian, V. 2001, ApJ, 557, 560

Pfrommer, C., \& Enßlin, T. A. 2004, A\&A, 426, 777

Rengelink, R. B., Tang, Y., de Bruyn, A. G., et al. 1997, A\&AS, 124, 259

Rybicki, G. B., \& Lightman, A. P. 1979, Radiative processes in astrophysics (New York: Wiley-Interscience)

Venturi, T., Giacintucci, S., Brunetti, G., et al. 2007, A\&A, 463, 937

Venturi, T., Giacintucci, S., Dallacasa, D., et al. 2008, A\&A, 484, 327 